\title{
Leptophilic fermion WIMP: Role of future lepton colliders
}

\author{
Shun-ichi Horigome, ${ }^{*}$ Taisuke Katayose, ${ }^{\dagger}$ Shigeki Matsumoto, ${ }^{*}$ and Ipsita Saha ${ }^{\S}$ \\ Kavli IPMU (WPI), UTIAS, University of Tokyo, Kashiwa, Chiba 277-8583, Japan
}

(Received 12 March 2021; accepted 4 August 2021; published 1 September 2021)

\begin{abstract}
The leptophilic weakly interacting massive particle (WIMP) is realized in a minimal renormalizable model scenario where scalar mediators with lepton number establish the WIMP interaction with the standard model leptons. We perform a comprehensive analysis for such a WIMP scenario for two distinct cases with an SU(2) doublet or singlet mediator, considering all of the relevant current theoretical, cosmological, and experimental constraints. We show that monophoton searches at near-future lepton collider experiments (ILC, FCC-ee, CEPC, etc.) can play a significant role in probing the yet unexplored parameter range allowed by the WIMP relic density constraint. This will complement the search prospects at the near-future hadron collider experiment HL-LHC. Furthermore, we discuss the combined model scenario including both the doublet and singlet mediators. The combined model is capable of explaining the long-standing muon $g-2$ anomaly, which is an additional advantage. We demonstrate that the region allowed by the latest muon $(g-2)$ result, which has been updated very recently at Fermi National Accelerator Laboratory, can also be probed at future colliders and thus will be a simultaneous authentication of the model scenario.
\end{abstract}

DOI: 10.1103/PhysRevD.104.055001

\section{INTRODUCTION}

A weakly interacting massive particle (WIMP) has been one of the most accepted particle candidates for dark matter $(\mathrm{DM})$ in the past few decades, as it can naturally explain the DM abundance in the current Universe through the standard thermal freeze-out mechanism [1,2]. The mass of a WIMP particle can vary between $\mathcal{O}(1) \mathrm{MeV}[3,4]$ and $\mathcal{O}(100) \mathrm{TeV}$ [5-14]. Specifically, a WIMP with mass of the order of the electroweak scale is the most compelling option, as a plethora of beyond-the-standard-model (BSM) scenarios-such as supersymmetric extensions, extra dimensions, etc.-provide a valid WIMP candidate along with solving the naturalness problem of electroweak symmetry breaking. In the general freeze-out scenario, the WIMP candidate is assumed to have interactions with the SM particles such that, in the early Universe at high temperature, the WIMP was in thermal equilibrium with all of the other primordial bath particles. However, the rate of interactions of the DM particle with the SM particles should

\footnotetext{
*shunichi.horigome@ipmu.jp taisuke.katayose@ipmu.jp

\$shigeki.matsumoto@ipmu.jp

\$ipsita.saha@ipmu.jp
}

Published by the American Physical Society under the terms of the Creative Commons Attribution 4.0 International license. Further distribution of this work must maintain attribution to the author(s) and the published article's title, journal citation, and DOI. Funded by SCOAP . be enough to explain the current abundance of the DM candidate in the present Universe, namely, the relic density.

On the other hand, such interactions become useful for probing the nature of WIMP DM at collider and underground (direct-detection) experiments. Current DM directdetection and collider experiments, however, rely mostly on the WIMP's interactions with the SM quarks, and if the WIMP predominantly interacts with the SM leptons, the current experimental efficiency is insufficient to probe such interactions. This kind of WIMP with dominant interactions with the SM leptons is generally called a "leptophilic WIMP" [15-20], and in this article we analyze aspects of such a leptophilic WIMP that interacts with the SM leptons via some mediator particles, utilizing minimal and renormalizable models.

In general, a WIMP can be classified in terms of its quantum numbers, i.e., its spin and weak isospin quantum numbers [21]. In our study, we consider a $\mathrm{SU}(2)_{L}$ singlet and spin-half Majorana fermion as our desired leptophilic WIMP particle, which is stabilized by an additional $Z_{2}$ symmetry. Then, it turns out that an additional new particle called the mediator must be introduced to have a renormalizable interaction between the WIMP and the SM leptons, and it must be bosonic in nature. In the minimal renormalizable extension of the SM, one can either have three generations of $\mathrm{SU}(2)_{L}$-doublet scalar mediators corresponding to the left-handed leptons, or three generations of $\mathrm{SU}(2)_{L}$-singlets corresponding to the right-handed SM leptons. The quantum numbers of the scalar mediators will follow that of their SM lepton partners, and these must be 
odd under the new $Z_{2}$ symmetry. It is to be mentioned that we only focus on lepton-flavor-conserving interactions of the DM particle.

In view of the above model specification, we first consider two individual model scenarios with either the doublet or singlet mediators, and perform a comprehensive analysis considering all robust theoretical, cosmological, and experimental constraints. The theoretical constraint involves the vacuum stability conditions on the scalar potential with the extra scalar mediators. We carefully discard the parameter space that gives an unstable vacuum by choosing correct scalar quartic couplings. On the other hand, the current experimental constraint includes the relic abundance of the WIMP measured by the Planck Collaboration and limits from the LEP-II and LHC run II experiments. First of all, the relic abundance of the leptophilic WIMP for the low mass region $\mathcal{O}(\mathrm{GeV})$ can be achieved with the proper tuning of WIMP-lepton Yukawa couplings. However, at larger WIMP masses of $\mathcal{O}(\mathrm{TeV})$, the coannihilation of the WIMP with the scalar mediators will be the key mechanism to obtain the correct relic abundance. In calculating the relic abundance, we properly take into account the effect of the SM thermodynamics in the early Universe [22,23]. It is to be noted that our scenario consists of a singlet Majorana WIMP and scalar mediators with lepton numbers which resemble the $R$-parity-conserving minimal supersymmetric scenario of the bino-slepton coannihilation. We thus include the current 95\% C.L. exclusion limit on the bino-slepton mass from LEP-II and the latest LHC $13 \mathrm{TeV}$ run-II results. Additionally, extra constraints coming from the Higgs-todiphoton decay mode and from the oblique $T$-parameter constraint (for the doublet mediator scenario) are also implemented.

In the next part, we discuss the future prospect of the leptophilic WIMP. Here, the future high-luminosity LHC (HL-LHC) experiment is expected to probe a part of the unexplored parameter region covering a nondegenerate and highly degenerate WIMP-mediator mass region. Thus, we consider future lepton collider experiments as a competent alternative that can probe a mildly degenerate mass region as well. We perform a detailed analysis for monophoton searches ${ }^{1}$ for WIMP pair production at the ILC experiment and explore the reach of the searches at 95\% C.L at the ILC-250 GeV. We find that the ILC can give a complementary detection prospect to the future HL-LHC experiment for such a leptophilic WIMP.

Finally, we discuss the combined model scenario where both the doublet and singlet scalar extensions to the leptophilic WIMP are studied. The model can be preferred over the others as it can also explain the discrepancy between theory and experiment in the anomalous muon magnetic moment. We find the allowed parameter space

\footnotetext{
${ }^{1}$ For mono-Higgs searches at the ILC experiment in a similar scenario, please refer to Ref. [24].
}

that explains both the muon $g-2$ anomaly and WIMP relic abundance. Finally, we perform the same monophoton search analysis for the combined model, and find that the ILC-250 GeV can validate the parameter space that can explain the muon $g-2$ anomaly. This is an interesting finding as the muon anomaly has persisted at the Fermilab [25] experiment, and thus the monophoton search at the ILC will provide an additional clue to the leptophilic WIMP.

The paper is arranged as follows. In Sec. II we describe the Lagrangian for the SM gauge singlet Majorana fermionic WIMP and its leptophilic interactions via the scalar mediators, explaining all relevant parameters of the potential. We address each mediator type in separate subsections. Next, in Sec. III we discuss all of the present relevant constraints, starting from the vacuum stability conditions, relic abundance condition, and the current constraints from the LEP and LHC experiments. We show the allowed parameter space for each model that satisfies all of the current constraints. In Sec. IV we analyze the current allowed parameter space at future colliders, with an emphasis on the monophoton searches at ILC $250 \mathrm{GeV}$. We discuss the complementarity between the future hadron and the lepton colliders. Finally, in Sec. V we discuss the combined model scenario consisting of both the doublet and singlet mediators. Here, we present the calculation for the anomalous muon magnetic moment and show the allowed parameter space capable of explaining the experimental anomaly. Additionally, we repeat the monophoton analysis at the ILC for the combined model and discuss the prospect of the simultaneous validation of the model parameter space at the ILC that also explains the anomaly. Last, in Sec. VI we summarize our findings and conclude.

\section{MINIMAL MODELS OF THE LEPTOPHILIC WIMP}

We consider minimal and renormalizable models to explore the interaction of a singlet Majorana fermion WIMP that only interacts with the SM leptons. In the simplest system composed of the WIMP and SM particles, no renormalizable interactions exist due to the presence of the SM gauge symmetry and the newly imposed $Z_{2}$ symmetry making the WIMP stable. The WIMP (SM particles) is charged odd (even) under the $Z_{2}$. Hence, an additional new particle, called a mediator, is introduced. Possible quantum numbers that allow the mediator to have a renormalizable interaction between the WIMP and SM leptons are as follows ${ }^{2}$ :

\footnotetext{
${ }^{2}$ All of the cases lead to the four-point effective interaction $\left(\bar{\chi} \gamma_{\mu} \gamma_{5} \chi\right)\left(\bar{\ell}_{i} \gamma^{\mu} \ell_{j}\right)$, with $\chi$ and $\ell_{i}$ denoting the Majorana WIMP and the SM leptons with generation index, if the mediator mass is larger enough than the WIMP mass and electroweak scale and integrated out from their original (renormalizable) theories.
} 


\begin{tabular}{lccccc}
\hline Mediator Type & Spin & $\mathrm{SU}(3)_{c}$ & $\mathrm{SU}(2)_{L}$ & $\mathrm{U}(1)_{Y}$ & $\mathrm{Z}_{2}$ \\
\hline Scalar & 0 & $\mathbf{1}$ & $\mathbf{1}$ & -1 & -1 \\
Scalar & 0 & $\mathbf{1}$ & $\mathbf{2}$ & $-1 / 2$ & -1 \\
Vector & 1 & $\mathbf{1}$ & $\mathbf{1}$ & -1 & -1 \\
Vector & 1 & $\mathbf{1}$ & $\mathbf{2}$ & $-1 / 2$ & -1 \\
Vector & 1 & $\mathbf{1}$ & $\mathbf{1}$ & 0 & +1 \\
\hline
\end{tabular}

A similar approach for constructing dark matter models considering the full SM gauge symmetry and renormalizability taking appropriate mediators into account has been first proposed in Ref. [26], and it has fixed various problems of dark matter effective field theories as well as dark matter simplified models that were widely used for a long time to interpret the data on dark matter searches at various experiments and observations. Though their study was mainly focused on interactions between dark matter and quarks, as also pointed out in the reference, it is straightforward to apply this approach to the leptophilic case.

Renormalizable models including a vector mediator are complicated in general, as they should contain the "Higgs mechanism" to make the mediator massive and a few new (chiral) fermions need to be introduced to make the models anomaly free $[27,28]$. We therefore focus on the models with scalar mediators in this article: the mediator particle is either a $\mathrm{SU}(2)$ scalar doublet with $\mathrm{U}(1)$ hypercharge $-1 / 2$ $\left(Q=T_{3}+Y\right)$ [called a left(-handed) mediator] or a $\mathrm{SU}(2)$ scalar singlet with $\mathrm{U}(1)$ hypercharge -1 [called a right(handed) mediator]. Analogous to the three generations of SM leptons, for each case there are three scalar mediators corresponding to each lepton flavor. For the sake of simplicity, we consider a flavor-universal scenario in this article and define the scalar masses by only one degenerate mass parameter. We discuss this further in the following sections.

Then, in the following sections we discuss the phenomenology of individual model perspectives with left or right mediators. The effect of introducing both types of mediators is also discussed in a later part of Sec. V, including our motivation.

\section{A. Left-mediator model}

The left mediator is a SU(2) scalar doublet that has charged and neutral components analogous to the lefthanded charged lepton and neutrino in the SM. The complete Lagrangian including the three generations of left mediators consists of two additional parts besides the usual SM Lagrangian $\left(\mathcal{L}_{\mathrm{SM}}\right)$ and the kinetic terms of the WIMP and the scalar mediators. These are, respectively, the interaction between the WIMP and the mediators (denoted by $\mathcal{L}_{\mathrm{DM} L}$ ) and the scalar potential (denoted by $V_{L}$ ) describing the self-interactions of the new scalar doublets and their interactions with the Higgs boson:

$$
\begin{aligned}
\mathcal{L}_{L} & =\mathcal{L}_{\mathrm{SM}}+\frac{1}{2} \bar{\chi}\left(i \not \supset-m_{\chi}\right) \chi+\left(D_{L}^{\mu} \tilde{L}_{i}\right)^{\dagger}\left(D_{L \mu} \tilde{L}_{i}\right)+\mathcal{L}_{\mathrm{DM} L}-V_{L}\left(H, \tilde{L}_{i}\right), \\
\mathcal{L}_{\mathrm{DM} L} & =-y_{L} \bar{L}_{i} \tilde{L}_{i} \chi+\text { H.c. }, \\
V_{L} & =m_{\tilde{L}}^{2}\left|\tilde{L}_{i}\right|^{2}+\frac{\lambda_{L}}{4}\left|\tilde{L}_{i}\right|^{4}+\lambda_{L H}\left|\tilde{L}_{i}\right|^{2}|H|^{2}+\lambda_{L H}^{\prime}\left(\tilde{L}_{i}^{\dagger} \tau^{a} \tilde{L}_{i}\right)\left(H^{\dagger} \tau^{a} H\right)+\left[\frac{\lambda_{L H}^{\prime \prime}}{4}\left(\tilde{L}_{i}^{\dagger} H^{c}\right)^{2}+\text { H.c. }\right],
\end{aligned}
$$

where a summation over the repeated indices is implicitly assumed, and the index $i$ spans lepton flavors $(e, \mu, \tau)$. Additionally, $\chi$ is the WIMP, $L_{i}$ is the SM lepton doublet, $H$ is the Higgs doublet $\left(H^{c} \equiv i \sigma_{2} H^{*}\right), \tilde{L}_{i}$ is the scalar mediator doublet whose quantum numbers match with $L_{i}, \tau^{a}$ is the Pauli matrix, and $D_{L}^{\mu}$ is the covariant derivative acting on $\tilde{L}_{i}$.

Since we are assuming lepton flavor universality, the Lagrangian parameters $y_{L}, m_{\tilde{L}}, \lambda_{L}, \lambda_{L H}, \lambda_{L H}^{\prime}$, and $\lambda_{L H}^{\prime \prime}$ are common in different flavors. To avoid sizable contributions to tiny neutrino masses we impose lepton number symmetry, or, to be more precise, the $B-L$ symmetry in the model. This is equivalent to assigning the lepton quantum number to the mediator particles, and thus terms proportional to $\lambda_{L H}^{\prime \prime}$ are prohibited. After electroweak symmetry breaking, with $v \simeq 246 \mathrm{GeV}$ being the vacuum expectation value of the Higgs field, the physical masses of each component of the scalar mediator doublets are given as

$$
\begin{aligned}
& m_{\tilde{e}_{L_{i}}}^{2}=\left(\lambda_{L H}+\lambda_{L H}^{\prime}\right) \frac{v^{2}}{2}+m_{\tilde{L}}^{2}, \\
& m_{\tilde{\nu}_{L_{i}}}^{2}=\left(\lambda_{L H}-\lambda_{L H}^{\prime}\right) \frac{v^{2}}{2}+m_{\tilde{L}}^{2},
\end{aligned}
$$

with $\tilde{L}_{i}=\left(\tilde{\nu}_{L_{i}}, \tilde{e}_{L_{i}}\right)^{T}$. The nomenclature of the physical masses are analogous to the superpartners of the leptons in supersymmetric standard models, namely, the sleptons.

\section{B. Right-mediator model}

Akin to the left-mediator model, one can write down the full Lagrangian for the right-mediator model, where instead of doublets the mediators are SU(2) singlets with the same quantum numbers as the right-handed SM charged leptons. The explicit form of the Lagrangian is 


$$
\begin{aligned}
\mathcal{L}_{R}= & \mathcal{L}_{\mathrm{SM}}+\frac{1}{2} \bar{\chi}\left(i \not \supset-m_{\chi}\right) \chi+\left(D_{R}^{\mu} \tilde{R}_{i}\right)^{\dagger}\left(D_{R \mu} \tilde{R}_{i}\right) \\
& +\mathcal{L}_{\mathrm{DM} R}-V_{R}\left(H, \tilde{R}_{i}\right), \\
\mathcal{L}_{\mathrm{DM} R}= & -y_{R} \bar{E}_{i} \tilde{R}_{i} \chi+\text { H.c. }, \\
V_{R}= & m_{\tilde{R}}^{2}\left|\tilde{R}_{i}\right|^{2}+\frac{\lambda_{R}}{4}\left|\tilde{R}_{i}\right|^{4}+\lambda_{R H}\left|\tilde{R}_{i}\right|^{2}|H|^{2},
\end{aligned}
$$

where $E_{i}$ is the SM charged lepton singlet, $\tilde{R}_{i}$ is the scalar mediator singlet whose quantum numbers resemble $E_{i}$, and $D_{R}^{\mu}$ is the covariant derivative acting on $\tilde{R}_{i}$. Here we also consider that all of the parameters $y_{R}, m_{\tilde{R}}$, $\lambda_{R}$, and $\lambda_{R H}$ are the same for different lepton flavors. Taking the same nomenclature as above, the physical masses of the right mediators are given by

$$
m_{\tilde{e}_{R_{i}}}^{2}=\lambda_{R H} \frac{v^{2}}{2}+m_{\tilde{R}}^{2}
$$

\section{PRESENT STATUS OF THE LEPTOPHILIC WIMP}

We discuss all relevant theoretical and experimental constraints imposed on the leptophilic models defined in the previous section, and figure out the present status of the models.

\section{A. Theoretical constraint}

Since we introduced new scalar fields in the leptophilic WIMP models as the mediators, the stability of the scalar potential has to be confirmed. The complete scalar potential for the left-mediator model includes the SM Higgs potential in addition to $V_{L}$, and it is given by

$$
V_{L}^{\text {Full }}\left(H, \tilde{L}_{i}\right)=\mu^{2}|H|^{2}+\frac{\lambda}{4}|H|^{4}+V_{L}\left(H, \tilde{L}_{i}\right),
$$

where the explicit form of the potential $V_{L}$ is given in Eq. (1). First, we obtain the following constraints because of the requirement that the potential is bounded from below:

$$
\lambda>0, \quad \lambda_{L}>0, \quad \sqrt{\lambda \lambda_{L}}>2\left(\left|\lambda_{L H}^{\prime}\right|-\lambda_{L H}\right) .
$$

Next, since the $Z_{2}$ symmetry should not be broken after electroweak symmetry breaking to make the WIMP stable even in the present Universe, the scalar mediators should not develop nonzero vacuum expectation values. Hence, we obtain the other constraint from the requirement that the masses in Eqs. (2) and (3) are positive with $v=\left(-4 \mu^{2} / \lambda\right)^{1 / 2}$ :

$$
-\lambda m_{\tilde{L}}^{2} /\left(2 \mu^{2}\right)>\left|\lambda_{L H}^{\prime}\right|-\lambda_{L H} .
$$

It is then possible to prove that our vacuum-namely, the potential minimum with the vacuum expectation value of the Higgs field being $\langle H\rangle=(0, v / \sqrt{2})^{T}$ and that of the scalar mediator being $\langle\tilde{L}\rangle=0$-becomes a global one when the constraints (7) and (8) are satisfied.

Similar to the above, the constraints for a stable vacuum in the right-mediator model are

$$
\begin{aligned}
\lambda & >0, \quad \lambda_{R}>0, \quad \sqrt{\lambda \lambda_{R}}>-2 \lambda_{R H}, \\
-\lambda m_{\tilde{R}}^{2} /\left(2 \mu^{2}\right) & >-\lambda_{R H},
\end{aligned}
$$

which also makes the stable vacuum the global minimum of the scalar potential.

\section{B. Astrophysical and cosmological constraints}

\section{Relic abundance}

In the early Universe, the WIMP was in thermal equilibrium with all of the SM particles residing in the thermal bath, and its abundance is determined by the socalled "freeze-out" mechanism. The thermal relic abundance of the WIMP can be theoretically estimated by solving the Boltzmann equation. On the other hand, the mean mass density of the cold dark matter content of the present Universe was measured by the Planck experiment, which observes the cosmic microwave background, as well as by other astrophysical observations [29]:

$$
\Omega h^{2}=0.120 \pm 0.001 \text {. }
$$

Weak-scale interactions for thermal WIMPs can easily obtain the correct relic abundance with a thermally averaged annihilation cross section into SM particles of $\langle\sigma v\rangle=\mathcal{O}(1) \mathrm{pb}$.

In our case, when the scalar mediators are much heavier than the WIMP, the leptophilic WIMP only annihilates into the SM leptons and contributes to the relic abundance, as shown in the top-left diagram in Fig. 1 for the left-mediator model. In this limit, the WIMP annihilation cross section only depends on the WIMP mass $\left(m_{\chi}\right)$, the mediator mass $\left(m_{\tilde{e}_{L_{i}}} \& m_{\tilde{\nu}_{L_{i}}}\right.$ or $\left.m_{\tilde{e}_{R_{i}}}\right)$, and the Yukawa coupling $\left(y_{L}\right.$ or $\left.y_{R}\right)$ for the left- or right-mediator model, respectively. On the other hand, when the mediator mass and the WIMP mass are degenerate within $10 \%$, the relic density is controlled by coannihilation processes [30]. The scalar quartic couplings between the mediators and the SM Higgs also become important in this limit for the relic density calculation, as shown by several diagrams in Fig. 1 for the left-mediator model. In our analysis, we scan over the model parameter space considering the limits that yield the correct relic abundance from both the WIMP self-annihilation and coannihilation processes. The relic density is calculated utilizing the code micrOMEGAs-v5 [31]. 


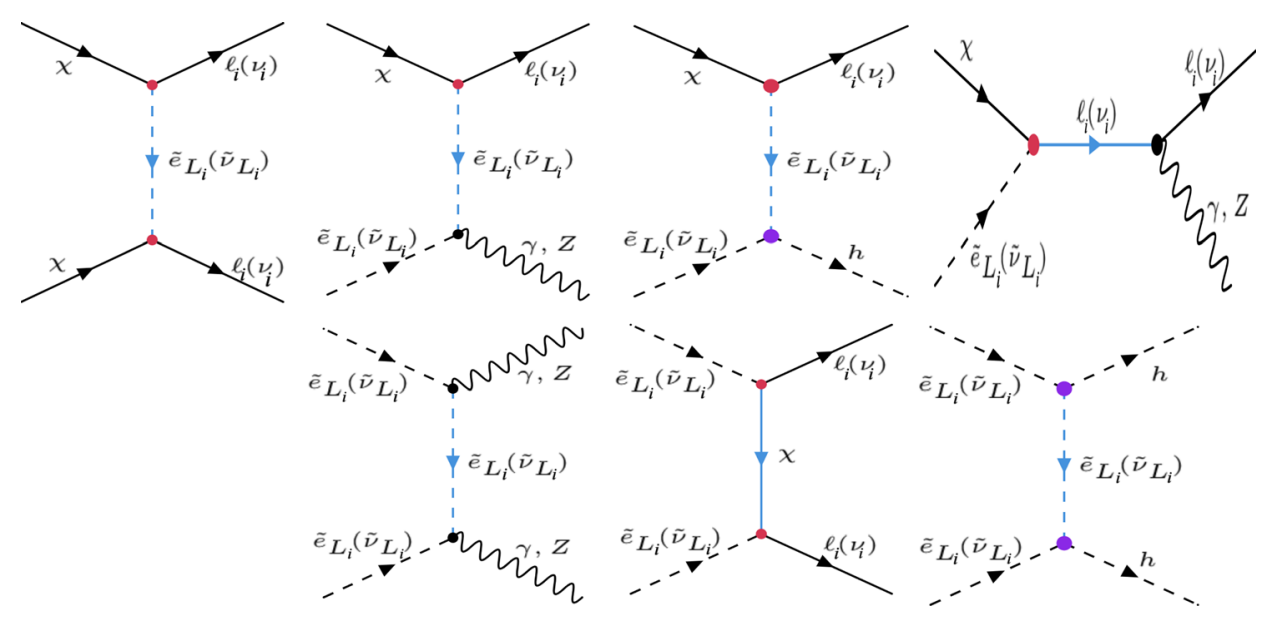

FIG. 1. Some illustrative Feynman diagrams for self-annihilation and coannihilation processes to calculate the relic abundance of the WIMP in the left-mediator model. Red vertices correspond to Yukawa coupling $y_{L}$, while purple vertices are proportional to the scalar coupling $\lambda_{L H}$. For the right-mediator model, $\tilde{e}_{L_{i}}$ should be replaced by $\tilde{e}_{R_{i}}$ and there is no neutral partner.

The uncertainty in the relic density calculation due to SM thermodynamics has recently been estimated $[22,23]$, where it was shown that the uncertainty of the effective massless degrees of freedom can be as large as $10 \%$, depending on the temperature of the Universe. This in turn induces an $\mathcal{O}(5 \%)$ uncertainty in the WIMP density during the freeze-out. We have incorporated this uncertainty in micrOMEGAs using the data provided in Ref. [23].

\section{Direct and indirect detections}

Direct dark matter detection is known to be a very powerful tool in searches for various WIMP candidates. The detection, however, relies on the (coherent) scattering between the WIMP and a nucleus, which is not efficient for leptophilic WIMPs. Here, one might think that such a scattering emerges radiatively through one-loop diagrams even in the leptophilic WIMP models we are discussing, where the SM leptons are propagating in the loop. The scattering cross section of such a process, however, turns out to be too small to detect at present and near-future detectors when the leptophilic WIMP is a Majorana fermion [32]. Hence, we do not include any constraints from direct WIMP detection in our analysis.

On the other hand, indirect dark matter detection is known to be a very useful tool in searches for WIMP candidates irrespective of their interactions, where the signal strength is proportional to the WIMP self-annihilation cross section. The annihilation cross section in the leptophilic models we are discussing is, however, velocity ( $p$-wave) suppressed due to the angular momentum and $C P$ conservation, and is insignificant in the present Universe. Therefore, indirect WIMP detection constraints are also irrelevant to our scenario.

\section{Collider constraints}

\section{Direct mediator production at $\mathrm{LHC}$ and LEP experiments}

It is difficult to directly probe the WIMP at current LHC experiments, since it is a gauge singlet and interacts only with the SM leptons. On the other hand, the mediator particles are charged under SM gauge interactions and accessible at both lepton and hadron colliders. To evaluate the constraints from collider experiments on the leptophilic models, we can directly use the limits relevant for supersymmetric particles. This is because the leptophilic models become the same as the ( $R$-parity-conserving) minimal supersymmetric model (MSSM), with the lightest and nextto-lightest supersymmetric particles being the bino and the (degenerate) sleptons, respectively, while other sparticles are decoupled, when we fix the parameters of the leptophilic models to be $y_{L}=g^{\prime} / \sqrt{2}, \quad \lambda_{L}=\left(g^{2}+g^{\prime 2}\right) / 2$, $\lambda_{L H}=g^{\prime 2} \cos (2 \beta), \quad \lambda_{L H}^{\prime}=g^{2} \cos (2 \beta) / 4, \quad \lambda_{L H}^{\prime \prime}=0, \quad$ and $y_{R}=\sqrt{2} g^{\prime}, \lambda_{R}=2 g^{\prime 2}$, and $\lambda_{R H}=-2 g^{\prime 2} \cos (2 \beta){ }^{3}$. Here, $g$ and $g^{\prime}$ are the $\mathrm{SU}(2)_{L}$ and $\mathrm{U}(1)_{Y}$ gauge couplings, respectively, while $\tan \beta$ is the ratio of the vacuum expectation values of the two Higgs doublets in the MSSM. On the other hand, the sleptons are produced by electroweak Drell-Yan processes at collider experiments and decay into the bino and a SM lepton with a $100 \%$ branching fraction. Hence, the signal strength does not depend on the Yukawa and scalar interactions addressed above, and we can directly use collider limits on the supersymmetric particles to evaluate collider constraints on the leptophilic models.

\footnotetext{
${ }^{3}$ The MSSM has two Higgs doublets that makes the model free from quantum anomaly. Therefore, to make a comparison between the MSSM and the leptophilic models, we take the decoupling limit in MSSM Higgs sector.
} 

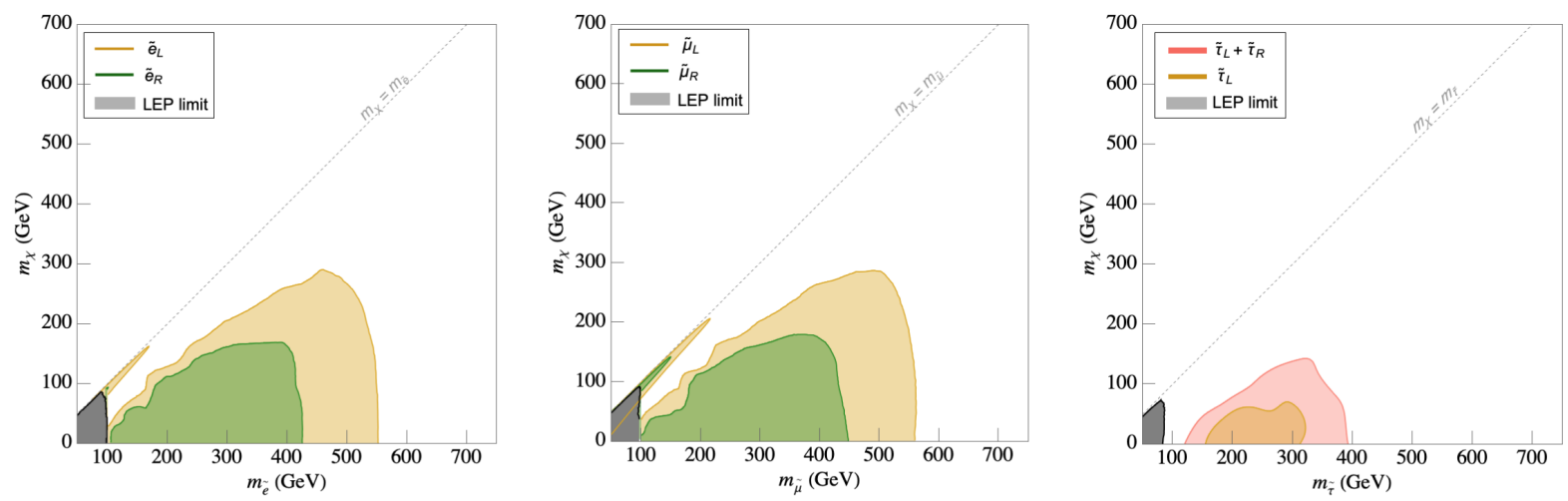

FIG. 2. 95\% C.L. exclusion (observed) limit on the mediator-WIMP mass plane obtained from the analysis of various slepton pair productions at the LHC (8 and $13 \mathrm{TeV}$ ) and LEP-II experiments.

The LEP experiment has searched for supersymmetric (electrically) charged sleptons, which decay dominantly to a charged SM lepton and a bino-like lightest supersymmetric particle (a Majorana fermion) [33]. As a result, the LEP experiment excluded the right-handed smuon, the super partner of the right-handed muon, with a mass below $94 \mathrm{GeV}$ for a neutralino-smuon mass gap above $10 \mathrm{GeV}$ [34]. This is a model-independent bound on leptonic charged scalars in our case, as mentioned above. We therefore impose this bound on both the right mediators and (as a conservative limit) the left mediators. The regions excluded by LEP for each charged slepton are also shown as the gray shaded areas in Fig. 2.

The LEP experiment has also searched for supersymmetric neutral sleptons, i.e., sneutrinos, where each sneutrino decays into a neutrino and the lightest neutralino. The model-independent bound on the mass of such a sneutrino is from the invisible decay width search for the $Z$ boson, as discussed in the last part of this subsection. On the other hand, it is also possible to search for sneutrinos using the monophoton channel caused by their pair production associated with a photon [35]. However, in our models, the DrellYan process via the $s$-channel exchange of the $Z$ boson is the only process that contributes to the monophoton signal, i.e., the other process via the $t$-channel exchange of the chargino does not contribute to the signal. As a result, no additional constraint on the sneutrino mass-which is more severe than the model-independent bound mentioned above-is obtained at the LEP experiment due to the smallness of the signal cross section [36]. Hence, we do not include the constraint obtained from the sneutrino pair production associated with a photon in our analysis.

The LHC experiments have also looked for such simplified scenarios where charged sleptons with a $100 \%$ branching ratio into their SM lepton partners and a WIMP (a bino-like lightest supersymmetric particle) can be produced. After LHC Run-2 at a center-of-mass energy of $13 \mathrm{TeV}$, the ATLAS Collaboration reported the 95\% exclusion limit on both left- and right-handed sleptons (selectrons and smuons) at $139 \mathrm{fb}^{-1}$ luminosity for a slepton-neutralino mass difference greater than $80 \mathrm{GeV}$ [37]. A dedicated search for compressed spectra where the slepton-neutralino mass difference is as low as $550 \mathrm{MeV}$ for a slepton mass around $70 \mathrm{GeV}$ was also performed, and the 95\% C.L. exclusion limit was presented [38]. In the left and middle panels of Fig. 2, we show this excluded region for selectron and smuon pair production as the yellow and green shaded regions for the left- and right-handed sleptons, respectively. The earlier limit from the $8 \mathrm{TeV} \mathrm{LHC}$ run is also included in the contours. For comparison purposes, we also show the excluded region for stau production (lefthanded stau production and left- + right-handed stau production) $[39,40]$ in the right panel of Fig. 2. As seen from the excluded region for the stau, the constraints from the stau searches are weaker than those of the selectron and smuon. Here, we would like to recall that we only consider the degenerate, flavor-universal case, and therefore we use the most sensitive smuon search limit on our model scenarios as the strongest bound.

Neutral sleptons, i.e., sneutrinos, have also been produced at the LHC by the electroweak Drell-Yan process, and the experiment is able to give some constraints on the sneutrinos if their decays produce enough visible particles [35]. The sneutrinos in our models, however, decay invisibly, i.e., each sneutrino decays into a neutrino and dark matter, with a $100 \%$ branching ratio, and one possible way to search for the sneutrinos is the so-called mono- $X$ search, with $X$ being a jet, photon, etc. Such signals have been intensively studied in Ref. [41], and no constraint stronger than that from the invisible $Z$-boson decay width search was obtained. Hence, we do not include such a constraint in our analysis.

\section{Radiative correction to the Higgs decay into diphoton}

With the accumulation of more data, the Higgs data at the LHC has been updated with unprecedented accuracy and it shows an increasing affinity for the SM value. In our model, the tree-level Higgs decay branching ratio will 

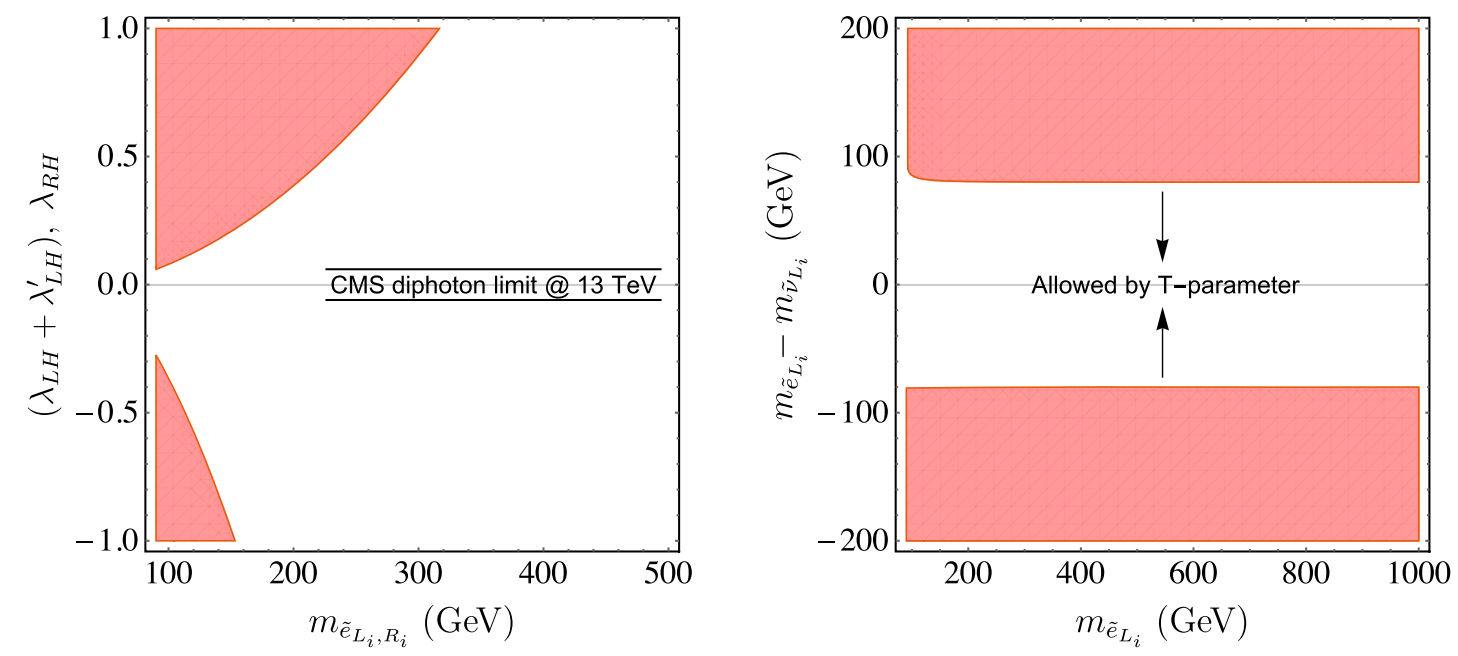

FIG. 3. The white region depicts the allowed parameter space at 95\% C.L. from the current Higgs-to-diphoton decay width measurement (left) and oblique $T$-parameter constraint (right).

exactly follow the SM value. However, the left and right charged mediator can significantly contribute to the loopinduced Higgs-to-diphoton decay [42-44]. The latest constraint on the Higgs-to-diphoton signal strength is given by the CMS Collaboration as $\mu_{\gamma \gamma}=1.18_{-0.14}^{+0.17}$ [45]. The signal strength is defined as the ratio of the Higgs production cross section times its branching ratio to the di-photon mode with the corresponding SM value. Since the exotic charged mediator does not contribute to the production channel and considering that the total decay width only changes negligibly due to the diphoton mode, the signal strength in our case can be approximated as the ratio of the partial decay width of the Higgs-to-diphoton decay and its SM value. Now, the charged mediator coupling to the SM Higgs is given by the scalar quartic couplings $\left(\lambda_{L H}+\lambda_{L H}^{\prime}\right)$ for the left mediator and $\lambda_{R H}$ for the right mediator [46]. In Fig. 3, we show the excluded parameter space at $95 \%$ C.L. in the charged mediator mass vs Higgs to charged mediator coupling plane as the red-shaded region.

\section{Electroweak precision measurements}

In the left-mediator case, the scalar mediators are doublets under the $\mathrm{SU}(2)_{L}$ gauge group. Therefore, they can significantly contribute to the self-energy of the SM electroweak gauge bosons, and an additional constraint from the socalled oblique $T$-parameter value that combines the electroweak precision data should be considered [47-49]. The contribution to the $T$ parameter from each generation of the left-mediator particles is given as follows [50]:

$$
\begin{aligned}
\Delta T_{\tilde{L}_{i}} & =\frac{1}{16 \pi^{2} \alpha v^{2}}\left(\frac{m_{\tilde{e}_{L_{i}}}^{2}+m_{\tilde{\nu}_{L_{i}}}^{2}}{2}-\frac{m_{\tilde{e}_{L_{i}}}^{2} m_{\tilde{\nu}_{L_{i}}}^{2}}{m_{\tilde{e}_{L_{i}}}^{2}-m_{\tilde{\nu}_{L_{i}}}^{2}} \log \frac{m_{\tilde{e}_{L_{i}}}^{2}}{m_{\tilde{\nu}_{L_{i}}}^{2}}\right) \\
& =\frac{\left(m_{\tilde{e}_{L_{i}}}-m_{\tilde{\nu}_{L_{i}}}\right)^{2}}{16 \pi^{2} \alpha v^{2}}\left[\frac{2}{3}-\frac{\left(m_{\tilde{e}_{L_{i}}}-m_{\tilde{\nu}_{L_{i}}}\right)^{2}}{30 m_{\tilde{\nu}_{L_{i}}}^{2}}+\mathcal{O}\left(\left\{\frac{m_{\tilde{e}_{L_{i}}}-m_{\tilde{\nu}_{L_{i}}}}{m_{\tilde{\nu}_{L_{i}}}}\right\}^{4}\right)\right],
\end{aligned}
$$

where $\alpha$ is the fine-structure constant. We see that, when the charged and neutral mediator particles are nearly degenerate in mass, the $T$ parameter depends not on their absolute masses but on the mass difference between them at leading order. Since we have three generations of degenerate scalar mediators, all of them will contribute to the $T$ parameter and put a stringent constraint on the mass splitting between the charged and neutral mediator particles. In the right panel of Fig. 3, we show the 95\% C.L. excluded region on the plane of charged mediator mass vs the mass gap between the charged mediator to the neutral mediator, where the latest value of the $T$-parameter constraint from new physics $\Delta T=0.05 \pm 0.06$ is used [34]. As seen in the figure, one can not get a mass gap larger than $80 \mathrm{GeV}$ for the charged scalar mass of interest.

\section{Invisible Higgs and Z-boson decays}

We have another constraint on the left mediators from precision Higgs and Z-boson invisible decay measurements. Although the LEP constraint demands that the charged components of the left mediators be at least greater 
than $90 \mathrm{GeV}$, there is no severe constraint on the mass of the neutral components. If the mass of neutral components is smaller than half of the Higgs or Z-boson mass, there is a decay channel such as $h \rightarrow \tilde{\nu}_{L_{i}} \tilde{\nu}_{L_{i}}^{*} \rightarrow \chi \chi \nu_{i} \bar{\nu}_{i}$ or $Z \rightarrow \tilde{\nu}_{L_{i}} \tilde{\nu}_{L_{i}}^{*} \rightarrow \chi \chi \nu_{i} \bar{\nu}_{i}$. These processes contribute to the invisible decay width of the Higgs or $Z$ boson as

$$
\begin{gathered}
\Gamma\left(h \rightarrow \tilde{\nu}_{L_{i}} \tilde{\nu}_{L_{i}}^{*}\right)=\frac{\left(\lambda_{L H}-\lambda_{L H}^{\prime}\right)^{2} v^{2}}{16 \pi m_{h}} \sqrt{1-\frac{4 m_{\tilde{\nu}_{L_{i}}}^{2}}{m_{h}^{2}},} \\
\Gamma\left(Z \rightarrow \tilde{\nu}_{L_{i}} \tilde{\nu}_{L_{i}}^{*}\right)=\frac{\alpha m_{Z}}{48 \sin ^{2} \theta_{W} \cos ^{2} \theta_{W}}\left(1-\frac{4 m_{\tilde{\nu}_{L_{i}}}^{2}}{m_{Z}^{2}}\right)^{3 / 2},
\end{gathered}
$$

where $m_{h}$ and $m_{Z}$ are the Higgs and Z-boson masses, respectively, $\alpha$ is the fine-structure constant, and $\theta_{W}$ is the Weinberg angle. The invisible decay width of the $Z$ boson is usually expressed using the effective neutrino number coupling to the $Z$ boson, and the current value from the LEP experiment is $N_{\nu}=2.9840 \pm 0.0082$ [51], which restricts the mass of the neutral component to be larger than half of the $Z$-boson mass $\left(m_{\tilde{\nu}_{L}} \geq m_{Z} / 2\right)$. On the other hand, the invisible decay width of the Higgs boson-or, in other words, the invisible decay branching fraction of the Higgs boson which is defined by

$$
\mathcal{B}_{\text {inv }}=\frac{3 \Gamma\left(h \rightarrow \tilde{\nu}_{L_{i}} \tilde{\nu}_{L_{i}}^{*}\right)}{\Gamma(h \rightarrow \mathrm{SMs})+3 \Gamma\left(h \rightarrow \tilde{\nu}_{L_{i}} \tilde{\nu}_{L_{i}}^{*}\right)},
$$

with $\Gamma(h \rightarrow \mathrm{SMs}) \simeq 4.1 \mathrm{MeV}$ [52]—is constrained to be $\mathcal{B}_{\text {inv }} \leq 0.13$ by the ATLAS Collaboration [53]. The constraints from invisible decays of Higgs and $Z$ bosons are summarized in Fig. 4 in the $\left(m_{\tilde{\nu}_{L_{i}}}, \lambda_{L H}\right)$ plane, and we include them in our analysis.

\section{Constraints from anomalous magnetic moments of leptons}

As we will discuss in more detail in Sec. VB, the leptophilic WIMP models inherently predict a contribution to anomalous magnetic moments of leptons, such as the muon and electron. At present, experimental data concerning the moments gives $[25,54,55]$

$$
\begin{gathered}
\Delta a_{\mu} \equiv a_{\mu}^{\mathrm{exp}}-a_{\mu}^{\mathrm{SM}}=251(59) \times 10^{-11}, \\
\Delta a_{e} \equiv a_{e}^{\mathrm{exp}}-a_{e}^{\mathrm{SM}}=-88(36) \times 10^{-14}
\end{gathered}
$$

in terms of the deviation from the SM prediction. As we will also show quantitatively in Sec. V B, both the left- and right-mediator models predict negligible contributions to the anomalous magnetic moments, i.e., they are smaller

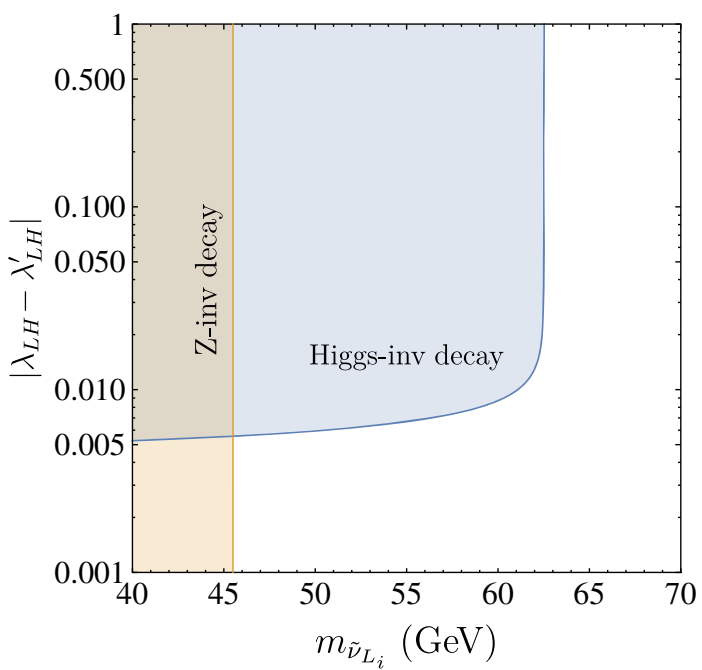

FIG. 4. Constrains on the Higgs invisible decay width from LHC(blue shaded) and on the Z-boson invisible decay width from LEP(orange shaded).

than the size of the errors shown above. This means that both models are unable to explain the anomalous magnetic moment of the muon (if we take the $4 \sigma$ discrepancy between the experimental result and the SM prediction seriously). On the other hand, the discrepancy for the anomalous magnetic moment of the electron is around the $2 \sigma$ level, and thus it should be used to constrain the models. However, the contributions to the moment of the electron from the models are negligibly small, so we do not have serious constraints from the $\Delta a_{e}$ result.

\section{E. Present status of the leptophilic WIMP}

Following all of the constraints in the previous subsections, we set the range of the scan for the model parameters. First of all, we require that the mediator mass be greater than the WIMP mass, i.e., $m_{\chi}<\left\{m_{\tilde{e}_{L_{i}}}, m_{\tilde{\nu}_{L_{i}}}, m_{\tilde{e}_{R_{i}}}\right\}$, and fix the quartic parameters $\lambda_{L}, \lambda_{R}=1$ which do not contribute to any physical observables and are only required to be positive from the vacuum stability conditions given in Sec. III A. The other relevant parameters are varied as

$$
\begin{array}{ll}
\left|\lambda_{L H}\right| \leq 1, \quad\left|y_{L}\right| \leq 1, & 90 \mathrm{GeV} \leq m_{\tilde{e}_{L_{i}}} \leq 2 \mathrm{TeV}, \\
\left|m_{\tilde{e}_{L_{i}}}-m_{\tilde{\nu}_{L_{i}}}\right| \leq 80 \mathrm{GeV}, & m_{\tilde{\nu}_{L_{i}}}>45 \mathrm{GeV}, \\
\left|\lambda_{R H}\right| \leq 1, \quad\left|y_{R}\right| \leq 1, & 90 \mathrm{GeV} \leq m_{\tilde{e}_{R_{i}}} \leq 2 \mathrm{TeV}, \\
1 \mathrm{GeV} \leq m_{\chi} \leq 2 \mathrm{TeV} . &
\end{array}
$$

Note that the mass gap between the two components of the $\mathrm{SU}(2)$ doublet in the left-mediator model takes care of the oblique $T$-parameter constraint in Fig. 3. For collider, Higgs-to-diphoton, and Higgs-to-invisible constraints, we use the $2 \sigma$ exclusion contours on our scanned 
parameter space in the allowed region of the correct relic abundance of the WIMP.

Next, we discuss our findings in detail. We scan our parameter space in the range given in Eq. (17), incorporating constraints on the scalar quartic couplings from Higgs-to-diphoton and Higgs-to-invisible width searches shown in Fig. 3 as well as the theoretical constraint in Sec. III A. The scanning is done with the help of the tool emcee [56] to make a proper sampling. The allowed parameters are then passed through the micrOMEGAs code to perform the relic abundance calculation. It is worth mentioning here that the experimental uncertainty of the dark matter relic abundance is much weaker than the uncertainty in the theoretical calculation that comes from the massless degrees of freedom in the early Universe. Therefore, to find the parameter space allowed by the relic abundance constraint, we perform a $\chi^{2}$ analysis considering the $2 \sigma$ uncertainty in the theoretical calculation and choose the central value at $\Omega h^{2}=0.120$ from the Planck experimental data.

In Fig. 5 we show the presently allowed parameter space in the $\left(m_{\tilde{e}_{L_{i}}\left(\tilde{e}_{R_{i}}\right)}, m_{\chi}\right)$ plane for the left-mediator (topleft panel) and right-mediator (top-right panel) models. The regions covered by green points are allowed by the present constraints at $95 \%$ C.L. It is evident from the figure that, at large WIMP mass, only degenerate masses for the WIMP and the mediators can satisfy the correct relic abundance via the coannihilation mechanism. In this coannihilating mass region, the scalar quartic coupling of
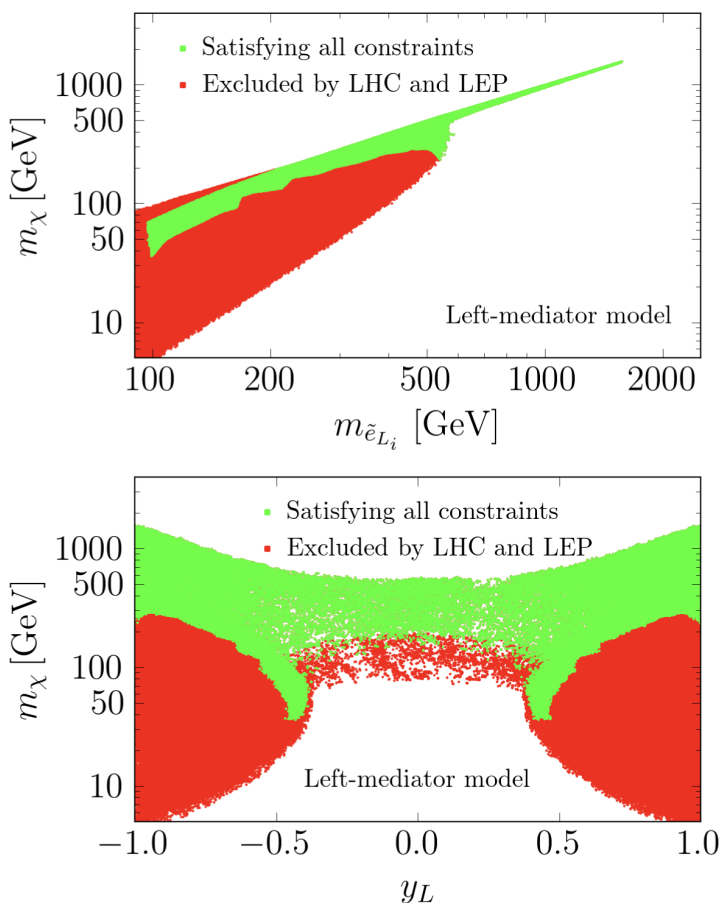

the mediators becomes effective and the correct relic abundance for the largest WIMP mass is achieved for the largest value of the scalar coupling $\lambda_{L H(R H)} \sim 1$, which renders an upper bound on the coannihilating mass range at 1.5 TeV. On the other hand, at small WIMP mass, the Yukawa coupling needs to be large enough to keep the annihilation cross section around $1 \mathrm{pb}$. This is because the WIMP and the mediators are still required to be (mildly) degenerate in mass, so that the annihilation cross section is proportional to $\sim y_{L}^{4}\left(y_{R}^{4}\right) / m_{\chi}^{2}$ (with $m_{\chi} \sim m_{\tilde{e}_{L_{i}}\left(\tilde{e}_{R_{i}}\right)}$ ) unless the coannihilation comes into play. This fact can also be seen in the bottom panels of Fig. 5, where the presently allowed parameter space in the $\left(y_{L}\left(y_{R}\right), m_{\chi}\right)$ plane is shown. In the left-mediator model, the coannihilation plays the dominant role when the WIMP mass is $m_{\chi}>500 \mathrm{GeV}$, while for the right-mediator case it appears beyond $400 \mathrm{GeV}$. The presence of an additional degree of freedom $\left(\tilde{\nu}_{L_{i}}\right)$ in the left mediator model allow heavier WIMPs of correct relic abundance obtained via the WIMP self-annihilation process.

Furthermore, the regions covered by red points in all of the panels of the figure depict the regions excluded only by the direct searches of the scalar mediators at LHC and LEP discussed in Sec. III A 1. As expected, a significant region with non-degenerate masses has already been excluded by the searches, while the degenerate mass region still survives that can be easily probed at the future lepton colliders, as discussed in the following section.
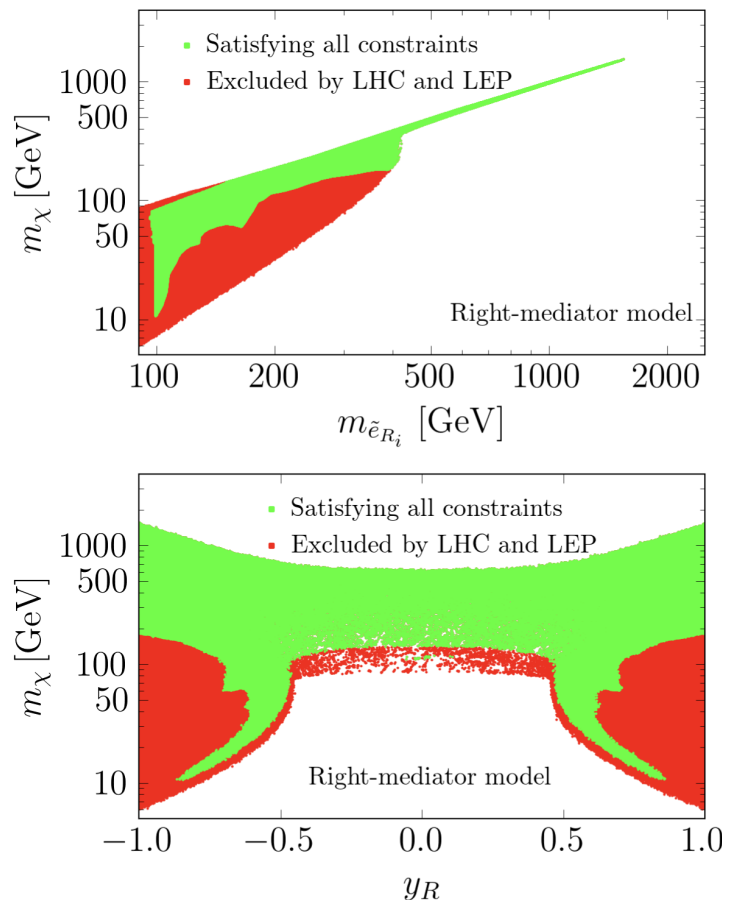

FIG. 5. Allowed parameter space at 95\% C.L. from theoretical and experimental constraints in the $\left(m_{\chi}, m_{\tilde{e}_{L_{i}}\left(\tilde{e}_{R_{i}}\right)}\right)$ plane (top panels) and $\left(y_{L}\left(y_{R}\right), m_{\chi}\right)$ plane (bottom panels) for the left-mediator (left panels) and right-mediator (right panels) models, respectively. See text for details. 

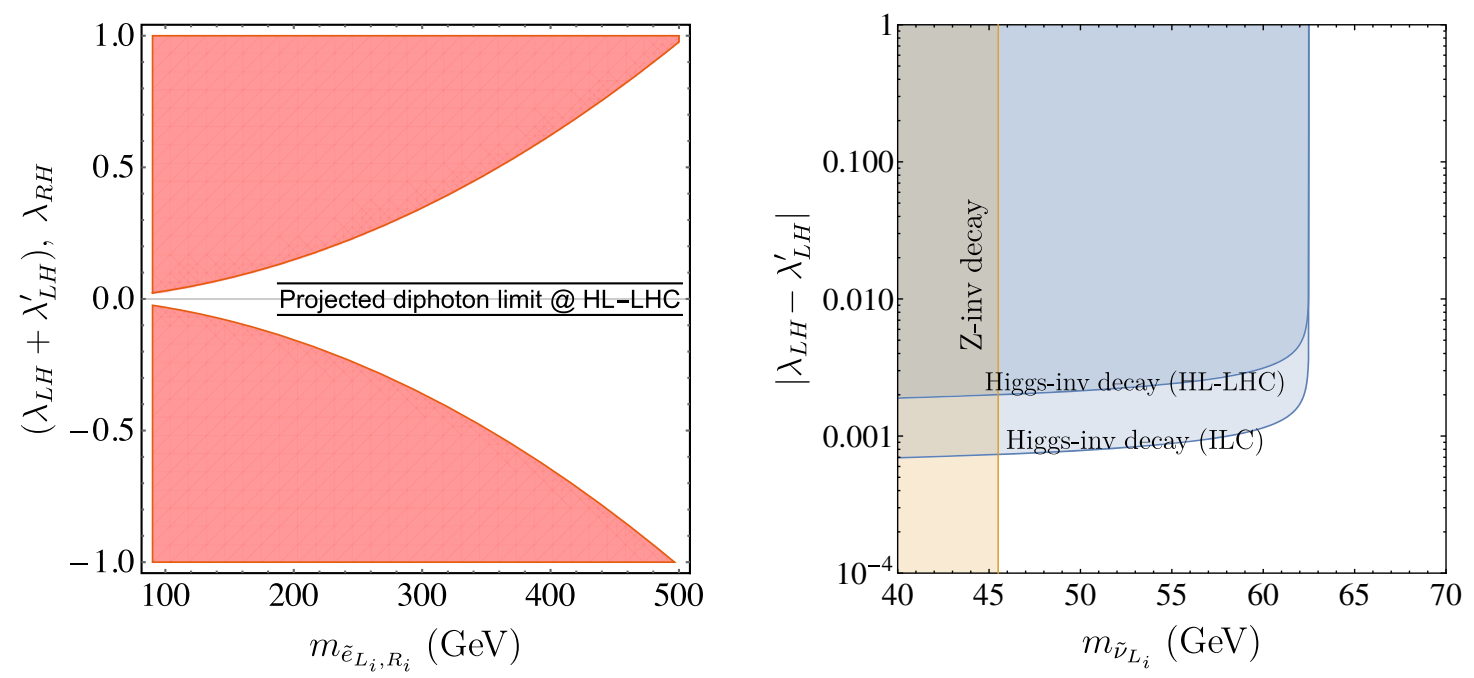

FIG. 6. The shaded region depicts the projected reach of parameter space at 95\% C.L. from the Higgs-to-diphoton (left) and Higgs-toinvisible (right) decay width measurements. See text for details.

\section{FUTURE PROSPECTS OF THE LEPTOPHILIC WIMP}

In this section we discuss the search sensitivity for the leptophilic WIMP at future collider experiments, focusing on the International Linear Collider (ILC), within the leftand right-mediator models, and figure out future prospects of the leptophilic WIMP search.

\section{A. Expected sensitivity at future colliders}

\section{Radiative correction to the Higgs decay into diphoton}

Future projections of the Higgs-to-diphoton decay mode predict that it will reach an accuracy of around $2 \%$ at the HL-LHC [57]. Therefore, the coupling of the charged scalar mediators to the Higgs will be further constrained. In Fig. 6 we show the projected sensitivity of the Higgs-todiphoton searches at the HL-LHC assuming that the uncertainty will be reduced to $2 \%$ while the central value is equal to the SM value.

\section{Invisible Higgs boson decay}

The measurement of the invisible Higgs decay width will be updated at the HL-LHC in the near future, and it is expected to be further improved at the ILC. Assuming that no new physics signals are observed at the measurements, the constraint on the branching fraction of the invisible Higgs decay width will be obtained as [58]

$$
\mathcal{B}_{\text {inv }} \leq \begin{cases}0.019 & {[\text { HL-LHC }]} \\ 0.0026 & {[250 \mathrm{GeV} \text { ILC }]}\end{cases}
$$

With these expected limits, the projected sensitivities of measuring the invisible Higgs decay width at the HL-LHC and ILC are shown in Fig. 6 in the $\left(m_{\tilde{\nu}_{L}}, \lambda_{L H}\right)$ plane.

\section{Direct mediator production at the HL-LHC}

No projected reaches have been reported by the HL-LHC working group for direct slepton pair production except for those of stau production [59], which are shown in Fig. 7 as blue and orange thin lines (95\% C.L. expected reach). These two lines (named cases 1 and 2) correspond to the results of two different analyses: one assumes that systematic uncertainties at the HL-LHC (14 TeV and $\left.3 \mathrm{ab}^{-1}\right)$ will decrease compared to the LHC Run-2 (case 1), while the other assumes that the uncertainties at the HL-LHC are the same as those in Run-2 (case 2). Future projected limits on selectron and smuon pair production are currently not available and they are expected to be stronger than the stau pair production, though it is not trivial how efficiently the searches cover the degenerate mass region between the mediator and dark matter.

\section{Direct mediator production at the ILC}

Direct pair production of charged mediators at the ILC was intensively studied in Ref. [60] within the context of supersymmetric (SUSY) models. Among various charged mediators, the production of mediators associated with the muon, i.e., smuon-type mediators, offers the most sensitive search for both the left- and right-mediator models. According to the reference, it turns out that a mediator mass less than $250 \mathrm{GeV}$, i.e., $m_{\tilde{e}_{L_{2} / R_{2}}} \leq 125 \mathrm{GeV}$, is covered at the ILC with $250 \mathrm{GeV}$ center-of-mass energy and $500 \mathrm{fb}^{-1}$ luminosity.

\section{Monophoton search at the ILC}

When the WIMP mass is lower than half of the centerof-mass energy at the ILC, the monophoton $(\gamma)$ channel works effectively because relatively large Yukawa couplings $y_{L / R}$ are required to achieve the observed relic 

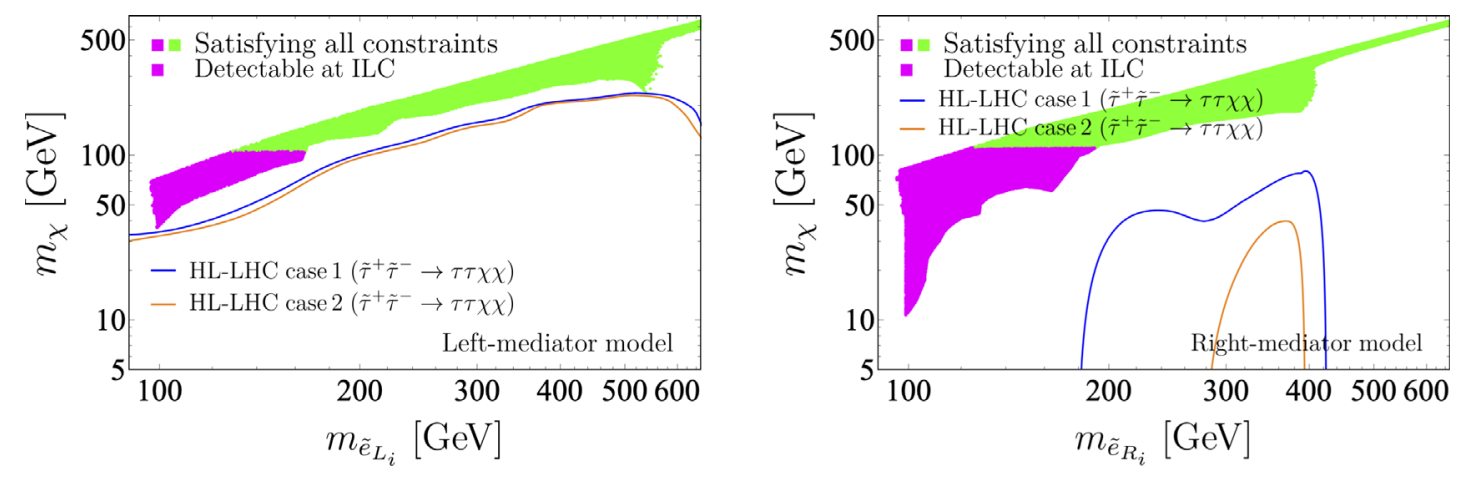

FIG. 7. The allowed parameter space for the left- and right-mediator models is shown in the left and right panels, respectively. The region covered by points is allowed by all of the constraints discussed in the previous section, while that covered by magenta points is the projected $95 \%$ C.L. reach at the $250 \mathrm{GeV}$ ILC taking both the mediator and monophoton searches into account. Blue and orange lines are the expected $95 \%$ C.L. reach of the direct stau pair production at the HL-LHC based on two assumptions concerning systematic uncertainties. See the text for more details.

density. However, when the WIMP and the mediators are degenerate, it becomes difficult to search for the WIMP using the mono- $\gamma$ channel because the couplings can be small due to the coannihilation mechanism. In such a case, we can use the direct pair production of charged mediators addressed above and the channel with pair creation of neutral mediator particles with an additional high-energy photon. In the latter case, the mediator will finally decay into a neutrino and a WIMP, and thus it can be counted as mono- $\gamma$ 's. The cross section of this channel is not suppressed by the Yukawa couplings because of the existence of the Drell-Yan process, and we will also have a severe constraint on the coannihilation region. The main background for the monophoton signal is due to the pair creation of neutrinos with an additional photon. We calculated the cross sections of the signal and the background for each energy bin by integrating analytic formulas of the differential cross sections. In order to suppress other backgrounds, we set the conditions $E_{\gamma} \geq 10 \mathrm{GeV}$ and $\cos \theta_{\gamma} \leq 0.98$, where $E_{\gamma}$ and $\theta_{\gamma}$ are the energy and polar angle of the photon, following a similar prescription to Ref. [61].

\section{B. Future prospects of the leptophilic WIMP}

We show the projected reach of the ILC in the mediatorWIMP mass plane for the left- and right-mediator models in the left and right panels of Fig. 7, respectively. The regions covered by green and magenta points are the parameter spaces allowed by all of the constraints discussed in the previous section, namely, they are the same as the regions covered by the green points in Fig. 5. On the other hand, the improvement of the Higgs-to-diphoton and Higgs-toinvisible decay width measurements at the HL-LHC and the ILC does not change the allowed parameter space of the leptophilic WIMP in the $\left(m_{\tilde{e}_{L_{i}}}, m_{\chi}\right)$ plane, and thus the benefit of the measurements for the WIMP search is not seen in the figure. The region covered by magenta points in the figure is the 95\% C.L expected reach of the ILC obtained by the search for direct charged mediator production and that of the monophoton. To perform the signalbackground analysis for the monophoton search, we followed the approach of our previous study [61]. ${ }^{4}$ First, we analytically calculated the differential cross sections of the signal and background processes $\left(e^{-} e^{+} \rightarrow \chi \chi \gamma, \tilde{\nu}_{L_{i}} \tilde{\nu}_{L_{i}}^{*} \gamma\right.$, and $\nu \bar{\nu} \gamma)$. Next, taking the initial-state radiation [63], beamstrahlung [64], and detector effects [65] into account, we numerically integrated the cross sections to obtain signal and background events in each energy bin with respect to the kinematical selection mentioned in Sec. IVA $4 .{ }^{5}$ Finally, we performed a simple likelihood analysis by comparing these signal and background predictions with the expected experimental data assuming $250 \mathrm{GeV}$ center-of-mass energy, $500 \mathrm{fb}^{-1}$ luminosity, and $0.1 \%$ systematic uncertainty. We did not utilize the polarization option of the ILC, i.e., we considered an unpolarized electron and positron collision, as the signal is strong enough and the sensitivity of the experiment to search for the leptophilic WIMP is determined by the center-of-mass energy of the collision and is almost irrespective of the polarization.

The figure shows that the projected reach of the leptophilic WIMP mass at 95\% C.L obtained by the monophoton search at the ILC is around $110 \mathrm{GeV}$. As we have shown in Sec. IV A 3, the HL-LHC is expected to have a good sensitivity to probe a larger mediator mass region which is not degenerate with the WIMP. On the other hand, in the presently allowed parameter space with the electroweak scale mediator, which is nothing but the region that the ILC will probe, we see more or less a degeneracy in mass between the mediator and the WIMP.

\footnotetext{
${ }^{4}$ Our approach reproduced the result of the analysis for the monophoton search at LEP [62] with good accuracy [61], justifying the approach to evaluate the expected sensitivity of the ILC.

${ }^{5}$ The bin width is set to $5 \mathrm{GeV}$ within the energy range of the emitted photon $E_{\gamma}$, with $E_{\gamma} \geq 10 \mathrm{GeV}$.
} 
The ILC and HL-LHC are thus expected to play complementary roles in searches for the leptophilic WIMP.

\section{COMBINED LEFT- AND RIGHT-HANDED MODEL}

In this section we discuss the consequences of considering the combined mediator scenario, namely, the leptophilic WIMP model with both the right- and left-handed mediators. After briefly addressing the minimal model (Lagrangian) and constraints on it, we discuss the motivation of the scenario and figure out the role of future colliders in testing the model.

\section{A. The minimal model}

\section{Lagrangian}

The renormalizable Lagrangian that contains both mediators is given as follows:

$$
\begin{aligned}
\mathcal{L}_{L R}= & \mathcal{L}_{\mathrm{SM}}+\frac{1}{2} \bar{\chi}\left(i \not \supset-m_{\chi}\right) \chi+\left(D_{L}^{\mu} \tilde{L}_{i}\right)^{\dagger}\left(D_{L \mu} \tilde{L}_{i}\right) \\
& +\left(D_{R}^{\mu} \tilde{R}_{i}\right)^{\dagger}\left(D_{R \mu} \tilde{R}_{i}\right)+\mathcal{L}_{\mathrm{DM}}-V_{L R}\left(H, \tilde{L}_{i}, \tilde{R}_{i}\right), \\
\mathcal{L}_{\mathrm{DM}}= & \mathcal{L}_{\mathrm{DM} L}+\mathcal{L}_{\mathrm{DM} R}, \\
V_{L R}= & V_{L}\left(H, \tilde{L}_{i}\right)+V_{R}\left(H, \tilde{R}_{i}\right)+\lambda_{L R}\left|\tilde{L}_{i}\right|^{2}\left|\tilde{R}_{i}\right|^{2} \\
& +\left(A m_{i} \tilde{L}_{i}^{\dagger} H \tilde{R}_{i}+\text { H.c. }\right) .
\end{aligned}
$$

$A$ is a dimensionless parameter contributing to the trilinear coupling and $m_{i}$ are the masses of the SM leptons $(e, \mu, \tau)$. This is a nontrivial assumption on the trilinear scalar couplings that is inspired by some relations from SUSY models or from models with minimal flavor violation $[66,67]$. As seen in the following discussion, it enables us to explain the experimental anomaly of the muon anomalous magnetic moment while avoiding the constraint from that of the electron in the combined model. Moreover, though the assumption makes the trilinear couplings suppressed, we can find the parameter region that satisfies the relic abundance condition and explains the muon $g-2$ anomaly, as we will also see later. In other words, if we relax the assumption, we will find a wider parameter region that explains both the dark matter relic abundance and the muon anomaly with heavier dark matter and mediator masses. Other parameters in the above Lagrangian are the same as those in Sec. II.

\section{Vacuum stability constraint}

After electroweak symmetry breaking, the $A$ term causes the mixing between $\tilde{L}_{i}$ and $\tilde{R}_{i}$. We scale this trilinear coupling by the lepton mass parameters as discussed in Sec. VA 1, so that it is most sensitive to the third-generation mixing. From the condition that the diagonalized mass of the mediators must be positive in our vacuum, we have

$$
\left|A v m_{\tau}\right| \leq \sqrt{2} m_{\tilde{e}_{L_{3}}} m_{\tilde{e}_{R_{3}}} .
$$

Remember the fact that the mediator masses $m_{\tilde{e}_{L_{i}}}$ and $m_{\tilde{e}_{R_{i}}}$ must be greater than the electroweak scale due to the collider constraints in Sec. III C 1, and thus the above constraint is always satisfied whenever the $A$ term is $\mathcal{O}(1-10)$. On the other hand, in order to guarantee the stability of our vacuum, we should confirm that the vacuum is the global minimum of the potential or its lifetime is longer than the age of the Universe. We have hence utilized the result in Ref. [68] to put a constraint on the $A$ term, where the condition of the vacuum stability is carefully investigated up to the oneloop level in the framework of the MSSM. ${ }^{6}$ According to the result, the constraint is given by $|A| \leq A_{c}$, with $A_{c} \simeq 80+0.3\left(m_{\tilde{e}_{L_{3}}} /[\mathrm{GeV}]\right)$, when $m_{\tilde{e}_{L_{3}}}=m_{\tilde{e}_{R_{3}}}$ and the dark matter is degenerate with the mediators in mass to comply with the surviving parameter region shown in Fig. 9. We thus adopt this constraint to guarantee the stability of our vacuum in the analysis of the combined model. ${ }^{7}$

\section{Relic abundance constraint}

As we have discussed above, we are interested in the parameter region where the WIMP and the mediators are at the electroweak scale; thus, for the sake of simplicity, we scan the model parameters assuming $m_{\tilde{e}_{L}}=m_{\tilde{e}_{R}}$ and $y_{R}^{4}=2 y_{L}^{4}$. The latter assumption is obtained by requiring $\sigma v(\chi \chi \rightarrow$ $\left.e_{R} \bar{e}_{R}\right)=\sigma v\left(\chi \chi \rightarrow e_{L} \bar{e}_{L}\right)+\sigma v\left(\chi \chi \rightarrow \nu_{L} \bar{\nu}_{L}\right)$, namely, both mediators contribute equally to the relic abundance of the WIMP when both mediators have the same mass, unless coannihilation processes come into play. This simplified analysis is enough to show the capability of future lepton colliders to search for the attractive parameter region of the combined model motivated by the muon $g-2$ anomaly as well as the dark matter abundance (thermal relic scenario). Uncertainties in the relic density calculation are taken into account in the same manner as those in Sec. III B 1.

\section{Other constraints}

Whenever the $A$ term is $\mathcal{O}(1-10)$, all other constraints discussed in the previous sections can be applied to the

\footnotetext{
${ }^{6}$ In order to make this vacuum stability constraint more accurate and also confirm the asymptotic safety of the combined model in Eq. (19), it is better to go beyond the one-loop calculation by taking into account the renormalization group running of the couplings (model parameters) [69,70]. On the other hand, the one-loop calculation is justified assuming the combined model is defined at an energy scale that is not far away from the electroweak scale and the coupling constants are below $\mathcal{O}(1)$, as we adopt in this paper.

${ }^{7}$ Honestly speaking, this constraint can be relaxed in the combined model defined in Eq. (19), as the quartic couplings of the mediators are taken to be large (without conflicting with any constraints discussed so far) in the combined model, while they are fixed by the gauge couplings in the MSSM. Note, however, that our discussion does not change even if we adopt the tighter constraint mentioned in the main text, as we are focusing on the parameter region that the future lepton collider experiments can access in their first stages.
} 
combined model to a good approximation. For the constraints coming from the measurements of the Higgs decay into diphoton, Higgs and Z-boson invisible decays, and also the electroweak precision measurement, the existence of the " $A$ " term affects the result subdominantly.For constraints from the direct mediator and WIMP production at present and future collider experiments, where the firstand second-generation mediators play an essential role in providing sensitive signals, the effect of the $A$ term is negligibly small. For those from branching fraction measurements of the Higgs decay into diphoton, Higgs and $Z$-boson invisible decays, and also the electroweak precision measurement, the existence of the $A$ term affects their signal strengths subdominantly. The direct darkmatter detection at underground experiments remain irrelevant to the leptophilic WIMP even for the combined model scenario. The signal strength of the indirect dark matter detection in astrophysical observations is slightly enhanced in the combined model scenario than that of the simplest left- and right-mediator models because of the $A$ term of $\mathcal{O}(10)$ compared to those in the simplest left- and rightmediator models. The sensitivity of the present observations is, however, still not enough to put a constraint on the combined model. We therefore take into account the constraints from the direct mediator and WIMP production measurements, the Higgs and Z-boson branching fractions, and the electroweak precision measurements at collider experiments based on those discussed in the previous sections in order to analyze the combined model.

\section{B. Motivation of the combined model}

An important motivation for going beyond the simplest scenarios with left or right mediators and going to the combined one is from the anomalous muon magnetic moment [20,71], namely, a long-standing discrepancy between the SM prediction and experimental results on the nature of the muon which may indicate the existence of new physics at the electroweak scale. It is also true that, as already mentioned at the beginning of Sec. II, it is obligatory to introduce two types of mediators regardless of the muon $g-2$ anomaly, as the left-handed and righthanded fermions are different in the quantum field theoretic aspect due to the chiral nature of the Lorentz symmetry in $(3+1)$ dimensional space-time. In other words, even if the muon $g-2$ anomaly disappears in the future, introducing the two types of mediators is motivated to interpret experimental data correctly [26].

Going back to the muon $g-2$ anomaly, the discrepancy between the experimental result and the SM prediction reaches the $4.2 \sigma$ level, and was very recently reported to be $\Delta a_{\mu} \equiv a_{\mu}^{\text {exp }}-a_{\mu}^{\mathrm{SM}}=251(59) \times 10^{-11}$ [25] at Fermilab. If the discrepancy persists even in future results from Fermilab [72] and J-PARC [73], it is expected to become a smoking-gun signature of new physics beyond the SM at a certain low energy scale.

Since the leptophilic dark matter couples to the SM leptons, it potentially contributes to the anomalous magnetic moments of the leptons. In the case of leptophilic dark matter with only left- or right-handed mediators, the contribution comes from the first two diagrams shown in Fig. 8. The explicit form of the contribution is given as follows [74]:

$$
\begin{aligned}
\Delta a_{\mu}^{\mathrm{L} / \mathrm{R}} & =-\frac{y_{L / R}^{2}}{96 \pi^{2}} \frac{m_{\mu}^{2}}{m_{\tilde{\mu}_{L / R}}^{2}} f(r) \\
& \simeq-10^{-10} y_{L / R}^{2}\left(\frac{100 \mathrm{GeV}}{m_{\tilde{\mu}_{L / R}}}\right)^{2} f(r), \\
f(r) & =\frac{1-6 r+3 r^{2}+2 r^{3}-6 r^{2} \log r}{(1-r)^{4}},
\end{aligned}
$$

where $\tilde{\mu}_{L / R} \equiv \tilde{e}_{L_{2} / R_{2}}, r \equiv m_{\chi}^{2} / m_{\tilde{\mu}_{L / R}}^{2}$, and $f(r)$ is a monotonically decreasing function taking a value between zero and one. It is hard to explain the anomaly due to the negative contribution.

On the contrary, the combined model with left-handed and right-handed mediators yields an additional contribution to the anomalous magnetic moment via the last two diagrams in Fig. 8. Using the mass insertion approximation, its explicit form is given as [75]

$$
\begin{aligned}
\Delta a_{\mu}^{\mathrm{L}+\mathrm{R}}= & -\frac{y_{L} y_{R}}{96 \pi^{2}} \frac{A v m_{\mu}^{2}}{\sqrt{2} m_{\chi}^{3}} f(x, y), \\
f(x, y)= & \frac{6}{x y}\left[\frac{-3+x+y+x y}{(x-1)^{2}(y-1)^{2}}+\frac{2 x \log x}{(x-y)(x-1)^{3}}\right. \\
& \left.+\frac{2 y \log y}{(y-x)(y-1)^{3}}\right],
\end{aligned}
$$

where $x \equiv m_{\tilde{\mu}_{L}}^{2} / m_{\chi}^{2}$ and $y \equiv m_{\tilde{\mu}_{R}}^{2} / m_{\chi}^{2}$. The function $f(x, y)$ is a monotonically decreasing function (along both the
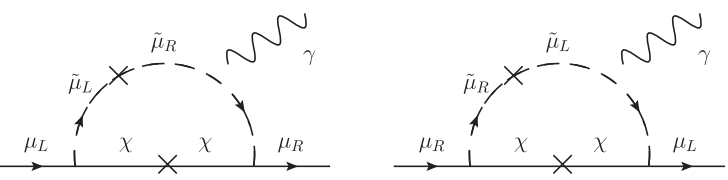

FIG. 8. Diagrams contributing to the anomalous muon magnetic moment. The first two diagrams are those from the simplest leptophilic WIMP model with the left-handed mediators. Similar diagrams for the right-mediator model can be found by swapping $L$ with $R$. The last two diagrams are from the leptophilic WIMP model with both left- and right-handed mediators, namely, the combined model. 
$x$ and $y$ directions) taking a value in between zero and one. The sign and size of the contribution $\Delta a_{\mu}^{\mathrm{L}+\mathrm{R}}$ can be properly tuned to account for the muon $g-2$ anomaly with an appropriate choice of sign and value of the Aterm.

In the limit of $x=y=1$, i.e., when the masses of the mediators and the DM are perfectly degenerate, $f(x, y)$ gets the maximum value of 1 , and the above contribution can be as large as $\Delta a_{\mu}^{\mathrm{L}+\mathrm{R}}=-\left[y_{L} y_{R} /\left(96 \pi^{2}\right)\right]\left[A v m_{\mu}^{2} /\left(\sqrt{2} m_{\chi}^{3}\right)\right] \simeq$ $-2 \times 10^{-9} y_{L} y_{R} A\left(100 \mathrm{GeV} / m_{\chi}\right)^{3}$. Hence, the muon $g-2$ anomaly can always be explained even if the $A$ term is $\mathcal{O}(1-10)$ whenever the dark matter as well as the mediators are at the electroweak scale. Conversely, this means that the electroweak scale WIMP and mediators, which are nothing but interesting targets during the first stage of future lepton colliders, require the size of the $A$ term to be $\mathcal{O}(1-10)$ to explain the muon $g-2$ anomaly.

Here, let us address the contribution of the leptophilic WIMP to the anomalous magnetic moment of the electron $\Delta a_{e}$. The contribution is given by the same formulas as above by replacing $m_{\mu}$ and $m_{\tilde{\mu}_{L / R}}=m_{\tilde{e}_{L_{2} / R_{2}}}$ with $m_{e}$ and $m_{\tilde{e}_{L_{1} / R_{1}}}$, respectively. As we have already mentioned in Sec. III D, the contribution is negligibly small within the left- and right-mediator models. In the combined model, the contribution is predicted to be $\Delta a_{e} \simeq 6 \times 10^{-14}$ when we fix the value of the $A$ parameter so that it can explain the muon $g-2$ anomaly. Comparing this contribution to the experimental data shown in Eq. (16), it turns out that its size is still within the error of the data. The observation of the anomalous magnetic moment of the electron hence does not put any severe constraints on the combined model.

\section{Present status and future prospects of the combined model}

The present status and future prospects of the combined leptophilic WIMP model are shown in left and right panels

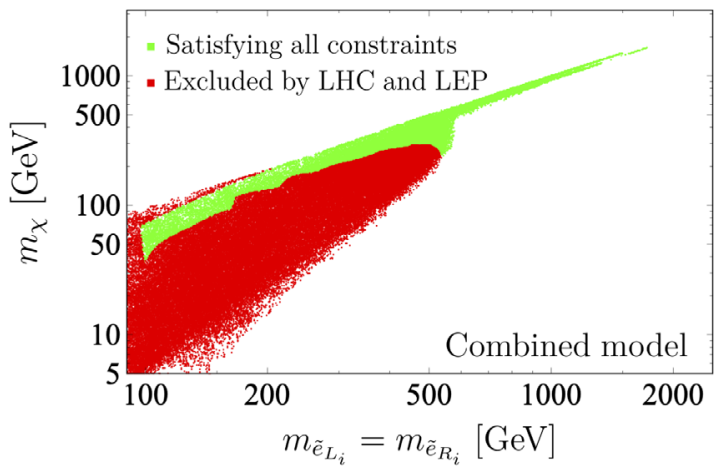

of Fig. 9, respectively, where the model parameters are scanned assuming $m_{\tilde{e}_{L}}=m_{\tilde{e}_{R}}$ and $y_{R}^{4}=2 y_{L}^{4}$ while imposing the constraints from the branching fraction measurements of Higgs and Z-boson decays and the electroweak precision measurements, as mentioned in Sec. VA. The color convention in the left panel is the same as that in Fig. 5. On the other hand, in the right panel the region covered by all of the points is the same as the one covered by green points in the left panel, with the color gradation indicating the value of the $A$ term to explain the muon $g-2$ anomaly $\left(\Delta a_{\mu}^{\mathrm{L} / \mathrm{R}}=251 \times 10^{-11}\right)$. Here, $A_{c}$ is defined in Sec. VA 2. We note that some gray points for the light mass region around $m_{\tilde{e}_{L_{i}}}=m_{\tilde{e}_{R_{i}}} \sim 300 \mathrm{GeV}$ correspond to the coannihilation of dark matter and sleptons, where $y_{L / R}$ can be very small. Moreover, the projected $95 \%$ C.L. reach by the searches for direct WIMP or mediator production at the ILC is also shown by the magenta solid line, while the expected 95\% C.L. reaches of direct stau pair production at the HL-LHC based on two assumptions concerning systematic uncertainties are shown by the blue and orange lines. (See Sec. IVA 3 for more detail.)

It is seen from the left panel of the figure that the allowed parameter region is similar to those of left- and righthanded mediator models discussed in the previous sections; the region with the large mass hierarchy between the WIMP and mediators and that with the extremely degenerated mass hierarchy among the particles are excluded by the present LHC data, and the region where these particles are mildly degenerated is presently allowed at the electroweak mass scale of the WIMP. On the other hand, in the right panel of the figure, the ILC is able to explore the region where the $A$ term is less than $\mathcal{O}(10)$, and it verifies our discussion in Sec. VA. We would like to further emphasize that such a range of scalar mixing preserves the stability of our vacuum, i.e., $|A|<A_{c}$, as discussed in Sec. VA 2. It is evident from Fig. 9 that the future lepton colliders such as

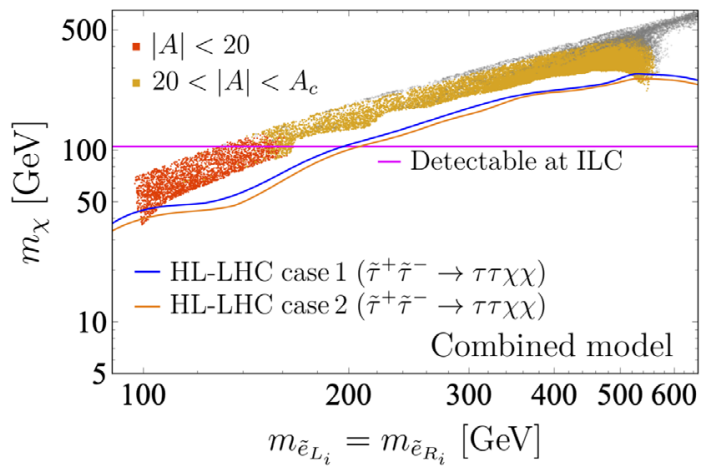

FIG. 9. Allowed parameter space at 95\% C.L. for the combined model. The region covered by green points in the left panel is allowed by all of the constraints discussed in Sec. VA, while the one covered by red points is excluded only by the present LHC and LEP experiments. On the other hand, the region covered by all of the points in the right panel is the same as the one covered by green points in the left panel, with the color gradation indicating the value of the $A$ term to explain the muon $g-2$ anomaly. $A_{c}$ is defined in Sec. VA 2 . Projected 95\% C.L. reaches by the search for direct mediator production at the ILC are also shown by the magenta line. Blue and orange lines are the expected $95 \%$ C.L. reaches of direct stau pair production at the HL-LHC based on two assumptions concerning systematic uncertainties. 
the ILC are expected to play a complementary role to the HL-LHC in the case of the leptophilic WIMP. It also serves the unique role in the search of WIMPs that can explain the muon $g-2$ while being consistent with the current dark matter density. ${ }^{8}$

\section{SUMMARY AND CONCLUSIONS}

Minimal and renormalizable models for a singlet Majorana fermion WIMP that interacts only with the SM leptons via the scalar mediators were considered. We performed an extensive analysis based on all current robust theoretical and experimental constraints at to show the feasibility of the models. To start with, we considered two distinct model scenarios: each has three generations of scalar mediators, but the mediators in the first scenario are doublets under $\mathrm{SM} \mathrm{SU}(2)_{L}$, while the other scenario has only singlet mediators under the $\mathrm{SU}(2)_{L}$ symmetry of the SM. Our choice of a Majorana fermion WIMP makes it necessary for the mediators to carry the exact same quantum numbers as their SM lepton partners. We considered the degenerate flavor-blind case for the sake of simplicity, and a single mass and coupling parameter will define all three generations of scalar mediators.

The most important constraint comes from the relic abundance of the WIMP. We performed a $\chi^{2}$ analysis to find the allowed parameter space at 95\% C.L. to the experimental limit, but including additional theoretical uncertainties that come from SM thermodynamics in the early Universe. The relic abundance constraint was further accompanied by a theoretical one (such as vacuum stability) and electroweak precision data and all relevant direct search limits from LEP and LHC Run-2. Our analysis showed that at large WIMP mass only the coannihilation mechanism with the mediator particle can survive the relic abundance constraint, and the largest allowed mass for the WIMP and mediator can be around $1.5 \mathrm{TeV}$. We also showed that the direct search limit on the scalar mediators only discards a part of the parameter space that contributes to the relic abundance via the self-annihilation region for a

\footnotetext{
${ }^{8}$ In addition to the HL-LHC, another experiment-the high-energy muon collider-was recently discussed to search for the parameter region with heavier dark matter and mediator particles [76,77].
}

WIMP mass below $300 \mathrm{GeV}$ and WIMP-mediator mass gap above $80 \mathrm{GeV}$.

As a next step, we discussed the possibility of probing the unexplored parameter region at future colliders, specifically at future lepton colliders. We performed a signalbackground analysis for the monophoton search at the ILC, and showed that the ILC-250 GeV can indeed pin down the WIMP mass around $110 \mathrm{GeV}$ with a mildly degenerate WIMP-mediator mass. As it is difficult to probe such a degenerate mass region at the HL-LHC, the ILC is expected to give a complementary search prospect for the leptophilic WIMP.

As the next-to-minimal extension, we also briefly considered the combined model scenario where both doublet and singlet mediators contribute to the relic abundance. The introduction of a third degree of freedom to the WIMP coannihilation decreases the largest allowed mass of the WIMP to $1.2 \mathrm{TeV}$. In the light of the anomalous magnetic moment of the muon, this combined model should be considered as the minimal scenario, since without the presence of both the mediator particles, it is impossible to explain the current discrepancy in the muon $g-2$. We showed that we have ample parameter space that can simultaneously explain both the relic density and the muon $g-2$ anomaly. Moreover, we repeated the ILC monophoton analysis for the combined model as well and found that the ILC-250 will be able to probe the aforementioned parameter space favored by the relic abundance and muon $g-2$ data. If the muon anomaly persists in future experimental data, the ILC will inevitably confirm or discard the model hypothesis.

\section{ACKNOWLEDGMENTS}

This work is supported by Grant-in-Aid for Scientific Research from the Ministry of Education, Culture, Sports, Science, and Technology (MEXT), Japan; 17H02878, 20H01895, 19H05810, 20H00153 (S. M.) and 18J21186 (S. H.), by World Premier International Research Center Initiative (WPI), MEXT, Japan (Kavli IPMU), and also by JSPS Core-to-Core Program (JPJSCCA20200002), and in part by the National Science Foundation (Grant No. NSF PHY-1748958) through the Kavli Institute for Theoretical Physics (KITP) program "New Physics from Precision at High Energies."
[1] J. Bernstein, L. S. Brown, and G. Feinberg, The cosmological heavy neutrino problem revisited, Phys. Rev. D 32, 3261 (1985).
[2] M. Srednicki, R. Watkins, and K. A. Olive, Calculations of relic densities in the early Universe, Nucl. Phys. B310, 693 (1988). 
[3] C. Boehm, T. A. Ensslin, and J. Silk, Can Annihilating dark matter be lighter than a few GeVs?, J. Phys. G 30, 279 (2004).

[4] C. Boehm, D. Hooper, J. Silk, M. Casse, and J. Paul, MeV Dark Matter: Has it Been Detected?, Phys. Rev. Lett. 92, 101301 (2004).

[5] K. Griest and M. Kamionkowski, Unitarity Limits on the Mass and Radius of Dark Matter Particles, Phys. Rev. Lett. 64, 615 (1990).

[6] K. Hamaguchi, S. Shirai, and T. T. Yanagida, Composite messenger baryon as a cold dark matter, Phys. Lett. B 654, 110 (2007).

[7] K. Hamaguchi, E. Nakamura, S. Shirai, and T. T. Yanagida, Decaying dark matter baryons in a composite messenger model, Phys. Lett. B 674, 299 (2009).

[8] K. Hamaguchi, E. Nakamura, S. Shirai, and T. T. Yanagida, Low-scale gauge mediation and composite messenger dark matter, J. High Energy Phys. 04 (2010) 119.

[9] H. Murayama and J. Shu, Topological dark matter, Phys. Lett. B 686, 162 (2010).

[10] T. Hambye and M. H. G. Tytgat, Confined hidden vector dark matter, Phys. Lett. B 683, 39 (2010).

[11] O. Antipin, M. Redi, and A. Strumia, Dynamical generation of the weak and Dark Matter scales from strong interactions, J. High Energy Phys. 01 (2015) 157.

[12] O. Antipin, M. Redi, A. Strumia, and E. Vigiani, Accidental composite dark matter, J. High Energy Phys. 07 (2015) 039.

[13] C. Gross, A. Mitridate, M. Redi, J. Smirnov, and A. Strumia, Cosmological abundance of colored relics, Phys. Rev. D 99, 016024 (2019).

[14] H. Fukuda, F. Luo, and S. Shirai, How heavy can neutralino dark matter be?, J. High Energy Phys. 04 (2019) 107.

[15] P. S. B. Dev, D. K. Ghosh, N. Okada, and I. Saha, Neutrino mass and Dark Matter in light of recent AMS-02 results, Phys. Rev. D 89, 095001 (2014),

[16] Y. Bai and J. Berger, Lepton portal dark matter, J. High Energy Phys. 08 (2014) 153.

[17] S. Chang, R. Edezhath, J. Hutchinson, and M. Luty, Leptophilic effective WIMPs, Phys. Rev. D 90, 015011 (2014).

[18] S. Junius, L. Lopez-Honorez, and A. Mariotti, A feeble window on leptophilic dark matter, J. High Energy Phys. 07 (2019) 136.

[19] S. Okawa and Y. Omura, Light mass window of lepton portal dark matter, J. High Energy Phys. 02 (2021) 231.

[20] J. Kawamura, S. Okawa, and Y. Omura, Current status and muon g-2 explanation of lepton portal dark matter, J. High Energy Phys. 08 (2020) 042.

[21] S. Banerjee, S. Matsumoto, K. Mukaida, and Y.-L. S. Tsai, WIMP Dark Matter in a Well-Tempered Regime: A case study on Singlet-Doublets Fermionic WIMP, J. High Energy Phys. 11 (2016) 070.

[22] K. Saikawa and S. Shirai, Primordial gravitational waves, precisely: The role of thermodynamics in the Standard Model, J. Cosmol. Astropart. Phys. 05 (2018) 035.

[23] K. Saikawa and S. Shirai, Precise WIMP dark matter abundance and standard model thermodynamics, J. Cosmol. Astropart. Phys. 08 (2020) 011.

[24] A. Jueid, S. Nasri, and R. Soualah, Searching for GeV-scale majorana dark matter: Inter spem et metum, J. High Energy Phys. 04 (2021) 012.
[25] B. Abi et al. (Muon g-2 Collaboration), Measurement of the Positive Muon Anomalous Magnetic Moment to 0.46 ppm, Phys. Rev. Lett. 126, 141801 (2021).

[26] P. Ko, A. Natale, M. Park, and H. Yokoya, Simplified DM models with the full SM gauge symmetry: The case of $t$-channel colored scalar mediators, J. High Energy Phys. 01 (2017) 086.

[27] E. Madge and P. Schwaller, Leptophilic dark matter from gauged lepton number: Phenomenology and gravitational wave signatures, J. High Energy Phys. 02 (2019) 048.

[28] C. Blanco, M. Escudero, D. Hooper, and S. J. Witte, Z' mediated WIMPs: Dead, dying, or soon to be detected?, J. Cosmol. Astropart. Phys. 11 (2019) 024.

[29] N. Aghanim et al. (Planck Collaboration), Planck 2018 results. VI. Cosmological parameters, Astron. Astrophys. 641, A6 (2020).

[30] K. Griest and D. Seckel, Three exceptions in the calculation of relic abundances, Phys. Rev. D 43, 3191 (1991).

[31] G. Bélanger, F. Boudjema, A. Goudelis, A. Pukhov, and B. Zaldivar, micromegas5.0: Freeze-in, Comput. Phys. Commun. 231, 173 (2018).

[32] M. J. Baker and A. Thamm, Leptonic WIMP coannihilation and the current dark matter search strategy, J. High Energy Phys. 10 (2018) 187.

[33] ALEPH, DELPHI, L3, OPAL Experiments Collaboration, http://lepsusy.web.cern.ch/lepsusy/.

[34] P. A. Zyla et al. (Particle Data Group Collaboration), Review of particle physics, Prog. Theor. Exp. Phys. 2020 (2020).

[35] P. Zyla et al. (Particle Data Group Collaboration), Review of particle physics, Prog. Theor. Exp. Phys. 2020, 083C01 (2020).

[36] A. Datta and A. Datta, Are light sneutrinos buried in LEP data?, Phys. Lett. B 578, 165 (2004).

[37] G. Aad et al. (ATLAS Collaboration), Search for electroweak production of charginos and sleptons decaying into final states with two leptons and missing transverse momentum in $\sqrt{s}=13 \mathrm{TeV} p p$ collisions using the ATLAS detector, Eur. Phys. J. C 80, 123 (2020),

[38] G. Aad et al. (ATLAS Collaboration), Searches for electroweak production of supersymmetric particles with compressed mass spectra in $\sqrt{s}=13 \mathrm{TeV} p p$ collisions with the ATLAS detector, Phys. Rev. D 101, 052005 (2020).

[39] G. Aad et al. (ATLAS Collaboration), Search for direct stau production in events with two hadronic $\tau$-leptons in $\sqrt{s}=13 \mathrm{TeV} p p$ collisions with the ATLAS detector, Phys. Rev. D 101, 032009 (2020).

[40] A. M. Sirunyan et al. (CMS Collaboration), Search for Supersymmetry with a Compressed Mass Spectrum in Events with a Soft $\tau$ Lepton, a Highly Energetic Jet, and Large Missing Transverse Momentum in Proton-Proton Collisions at $\sqrt{s}=\mathrm{TeV}$, Phys. Rev. Lett. 124, 041803 (2020).

[41] J. Ellis, A. Fowlie, L. Marzola, and M. Raidal, Statistical analyses of Higgs- and Z-portal Dark Matter models, Phys. Rev. D 97, 115014 (2018).

[42] J. F. Gunion, H. E. Haber, G. L. Kane, and S. Dawson, The Higgs Hunter's Guide (Perseus Publishing, Cambridge, 2000), Vol. 80. 
[43] A. Djouadi, The Anatomy of electro-weak symmetry breaking. I: The Higgs boson in the standard model, Phys. Rep. 457, 1 (2008).

[44] A. Djouadi, The Anatomy of electro-weak symmetry breaking. II. The Higgs bosons in the minimal supersymmetric model, Phys. Rep. 459, 1 (2008).

[45] A. Sirunyan et al. (CMS Collaboration), Measurements of Higgs boson properties in the diphoton decay channel in proton-proton collisions at $\sqrt{s}=13 \mathrm{TeV}$, J. High Energy Phys. 11 (2018) 185.

[46] A. Djouadi, V. Driesen, W. Hollik, and J. I. Illana, The Coupling of the lightest SUSY Higgs boson to two photons in the decoupling regime, Eur. Phys. J. C 1, 149 (1998).

[47] H.-J. He, N. Polonsky, and S.-f. Su, Extra families, Higgs spectrum and oblique corrections, Phys. Rev. D 64, 053004 (2001).

[48] W. Grimus, L. Lavoura, O. Ogreid, and P. Osland, A precision constraint on multi-Higgs-doublet models, J. Phys. G 35, 075001 (2008).

[49] W. Grimus, L. Lavoura, O. Ogreid, and P. Osland, The oblique parameters in multi-Higgs-doublet models, Nucl. Phys. B801, 81 (2008).

[50] R. Barbieri, L. J. Hall, and V.S. Rychkov, Improved naturalness with a heavy Higgs: An Alternative road to LHC physics, Phys. Rev. D 74, 015007 (2006).

[51] S. Schael et al. (ALEPH, DELPHI, L3, OPAL, SLD, LEP Electroweak Working Group, SLD Electroweak Group, SLD Heavy Flavour Group Collaborations), Precision electroweak measurements on the $Z$ resonance, Phys. Rep. 427, 257 (2006).

[52] D. M. Asner et al., ILC Higgs white paper, in Community Summer Study 2013: Snowmass on the Mississippi (2013), http://cds.cern.ch/record/1605366.

[53] ATLAS Collaboration, Search for invisible Higgs boson decays with vector boson fusion signatures with the ATLAS detector using an integrated luminosity of $139 \mathrm{fb}^{-1}$ (2020) http://cds.cern.ch/record/2715447.

[54] D. Hanneke, S. Fogwell, and G. Gabrielse, New Measurement of the Electron Magnetic Moment and the Fine Structure Constant, Phys. Rev. Lett. 100, 120801 (2008).

[55] R. H. Parker, C. Yu, W. Zhong, B. Estey, and H. Müller, Measurement of the fine-structure constant as a test of the standard model, Science 360, 191 (2018).

[56] D. Foreman-Mackey, D. W. Hogg, D. Lang, and J. Goodman, emcee: The MCMC Hammer, Publ. Astron. Soc. Pac. 125, 306 (2013).

[57] M. Cepeda et al., Report from Working Group 2: Higgs Physics at the HL-LHC and HE-LHC, CERN Yellow Rep. Monogr. 7, 221 (2019).

[58] J. de Blas et al., Higgs boson studies at future particle colliders, J. High Energy Phys. 01 (2020) 139.

[59] ATLAS Collaboration, Prospects for searches for staus, charginos and neutralinos at the high luminosity LHC with the ATLAS Detector, http://cds.cern.ch/record/2651927.
[60] S. Baum, P. Sandick, and P. Stengel, Hunting for scalar lepton partners at future electron colliders, Phys. Rev. D 102, 015026 (2020).

[61] D. K. Ghosh, T. Katayose, S. Matsumoto, I. Saha, S. Shirai, and T. Tanabe, Role of future lepton colliders for fermionic Z-portal dark matter models, Phys. Rev. D 101, 015007 (2020).

[62] P. J. Fox, R. Harnik, J. Kopp, and Y. Tsai, LEP shines light on dark matter, Phys. Rev. D 84, 014028 (2011).

[63] E. A. Kuraev and V. S. Fadin, On radiative corrections to e+ e- single photon annihilation at high-energy, Sov. J. Nucl. Phys. 41, 466 (1985).

[64] A. K. Datta, K. Kong, and K. T. Matchev, The impact of beamstrahlung on precision measurements at CLIC, eConf C050318, 0215 (2005).

[65] T. Behnke, J. E. Brau, B. Foster, J. Fuster, M. Harrison, J. M. Paterson et al., The international linear collider technical design report—volume 1: Executive summary, arXiv:1306 .6327.

[66] G. D’Ambrosio, G. F. Giudice, G. Isidori, and A. Strumia, Minimal flavor violation: An effective field theory approach, Nucl. Phys. B645, 155 (2002).

[67] G. Colangelo, E. Nikolidakis, and C. Smith, Supersymmetric models with minimal flavour violation and their running, Eur. Phys. J. C 59, 75 (2009).

[68] G. H. Duan, C. Han, B. Peng, L. Wu, and J. M. Yang, Vacuum stability in stau-neutralino coannihilation in MSSM, Phys. Lett. B 788, 475 (2019).

[69] G. Hiller, C. Hormigos-Feliu, D. F. Litim, and T. Steudtner, Anomalous magnetic moments from asymptotic safety, Phys. Rev. D 102, 071901 (2020).

[70] G. Hiller, C. Hormigos-Feliu, D. F. Litim, and T. Steudtner, Model building from asymptotic safety with Higgs and flavor portals, Phys. Rev. D 102, 095023 (2020).

[71] L. Calibbi, R. Ziegler, and J. Zupan, Minimal models for dark matter and the muon g-2 anomaly, J. High Energy Phys. 07 (2018) 046.

[72] J. Grange et al. (Muon g-2 Collaboration), Muon (g-2) Technical Design Report, arXiv:1501.06858.

[73] H. Iinuma (J-PARC muon g-2/EDM Collaboration), New approach to the muon g-2 and EDM experiment at J-PARC, J. Phys. Conf. Ser. 295, 012032 (2011).

[74] P. Agrawal, Z. Chacko, and C. B. Verhaaren, Leptophilic dark matter and the anomalous magnetic moment of the muon, J. High Energy Phys. 08 (2014) 147.

[75] T. Moroi, The Muon anomalous magnetic dipole moment in the minimal supersymmetric standard model, Phys. Rev. D 53, 6565 (1996).

[76] R. Capdevilla, D. Curtin, Y. Kahn, and G. Krnjaic, A guaranteed discovery at future muon colliders, Phys. Rev. D 103, 075028 (2021).

[77] R. Capdevilla, D. Curtin, Y. Kahn, and G. Krnjaic, A nolose theorem for discovering the new physics of $(g-2)_{\mu}$ at muon colliders, arXiv:2101.10334. 